\title{
Efficient and Stable Exponential Time Differencing Runge-Kutta Methods for Phase Field Elastic Bending Energy Models
}

\author{
Xiaoqiang Wang ${ }^{\mathrm{a}}$, Lili Ju ${ }^{\mathrm{b}, *}$, Qiang $\mathrm{Du}^{\mathrm{c}}$ \\ ${ }^{a}$ Department of Scientific Computing, Florida State University, Tallahassee, FL 32306, USA. \\ ${ }^{b}$ Department of Mathematics, University of South Carolina, Columbia, SC 29208, USA. \\ ${ }^{c}$ Department of Applied Physics and Applied Mathematics, Columbia University, NY 10027, USA.
}

\begin{abstract}
The Willmore flow formulated by phase field dynamics based on the elastic bending energy model has been widely used to describe the shape transformation of biological lipid vesicles. In this paper, we develop and investigate some efficient and stable numerical methods for simulating the unconstrained phase field Willmore dynamics and the phase field Willmore dynamics with fixed volume and surface area constraints. The proposed methods can be high-order accurate and are completely explicit in nature, by combining exponential time differencing Runge-Kutta approximations for time integration with spectral discretizations for spatial operators on regular meshes. We also incorporate novel linear operator splitting techniques into the numerical schemes to improve the discrete energy stability. In order to avoid extra numerical instability brought by use of large penalty parameters in solving the constrained phase field Willmore dynamics problem, a modified augmented Lagrange multiplier approach is proposed and adopted. Various numerical experiments are performed to demonstrate accuracy and stability of the proposed methods.
\end{abstract}

Keywords: Elastic bending energy, Willmore flow, phase field method, exponential time differencing, Runge-Kutta methods, augmented Lagrange multiplier

2010 MSC: 65M70, 65L06, 65Y20, 92C17

\section{Introduction}

The application of phase field methods to biological microstructures, especially in the modeling and simulations of cell membranes, has emerged in recent years $[14,10,11,16]$. Lipids, known as fat, are ubiquitous in biological systems. Over $70 \%$ of cell membranes are composed of lipids with a bilayer structure. The shape transformations of lipid microstructures, including budding and fusion, are closely associated with cell functions. A continuum bending elasticity model, first studied by Canham, Evans and Helfrich [3, 22, 27, 4] for a single-phase membrane is that the shape of a lipid membrane, is determined by the so-called sharp interface elastic bending energy. In the simplest setting (by ignoring the effect of spontaneous curvature and the contributions of

\footnotetext{
${ }^{2}$ L. Ju's research is partially supported by US National Science Foundation under grant numbers DMS-1521965. Q. Du's research is partially supported by US National Science Foundation under grant number DMS-1318586.

${ }^{*}$ Corresponding author $\mathrm{Du})$

Email addresses: wwang3@fsu.edu (Xiaoqiang Wang), ju@math.sc.edu (Lili Ju), qd2125@columbia.edu (Qiang
} 
the Gaussian curvature), the bending energy is given by the mean curvature energy

$$
\mathcal{E}=\int_{\Gamma} H^{2} d S
$$

where $H$ is the mean curvature of the membrane surface $\Gamma$. Geometrically, the problem of finding the surface $\Gamma$ that minimizes the mean curvature energy (1) is the so-called Willmore's problem [41]. The surface evolution driven by the gradient flow of (1) is called the Willmore flow.

During the past decade, phase field methods have been successfully developed for this elastic bending energy model for the lipid vesicles, see $[9,14,11,10,16,29,13,21]$. It is able to track a deforming interface without putting meshes on the surface, thus giving an easy treatment of topological changes of the interface $[15,12]$. Phase field methods also have been extensively used to study multi-component vesicles [40,35, 24], vesicle-substrate adhesion[44, 25], and vesicle-vesicle adhesion problems [26], dynamics of vesicles in fluids [13, 38, 23]. In the phase field approach, the membrane of a single vesicle can be visualized by the level set $\{\mathbf{x}: \phi(\mathbf{x})=0\}$ of a phase field function $\phi$ defined on a computational domain $\Omega$, where $\{\mathbf{x}: \phi(\mathbf{x})>0\}$ represents the inside of the membrane and $\{\mathbf{x}: \phi(\mathbf{x}) \leq 0\}$ the outside.

For the mean curvature energy (1), following [14], we may define the following modified elastic bending energy based on the phase field function $\phi$ :

$$
W(\phi)=\int_{\Omega} \frac{\epsilon}{2}\left|\Delta \phi-\frac{1}{\epsilon^{2}}\left(\phi^{2}-1\right) \phi\right|^{2} d \mathbf{x}
$$

where $\epsilon$ is a small positive parameter characterizing the width of diffusive interface or transition layer. Through asymptotic analysis [10,39], the consistency between the phase field formulation and the original sharp interface problem has been established as

$$
W(\phi) \sim \frac{4 \sqrt{2}}{3} \int_{\Gamma} H^{2} d S .
$$

In particular, in the sharp interface limit as $\epsilon \rightarrow 0$, the zero-level set of phase field function $\phi$ that minimizes (2), recovers the surface $\Gamma$ that minimizes the (1). In recent years, some progresses $[37,36,1]$ have been made towards the rigorous proof of the $\Gamma$-Convergence of the phase field functional $W(\phi)$ to the Willmore problem (1), which is closely related to the well-known De Giorgi conjecture [7].

The following evolution equation can be used to describe the phase field Willmore dynamics of the elastic bending energy $W(\phi)$ :

$$
\begin{aligned}
\frac{\partial \phi}{\partial t} & =-\frac{\delta W(\phi)}{\delta \phi} \\
& =-\epsilon \Delta f(\phi)+\frac{1}{\epsilon}\left(3 \phi^{2}-1\right) f(\phi)
\end{aligned}
$$

where $f(\phi)=\Delta \phi-\frac{1}{\epsilon^{2}}\left(\phi^{3}-\phi\right)$. This equation can be viewed as the Allen-Cahn type dynamics associated with the energy (2). The stable steady states of (3) give us solutions of the phased field Willmore's problem

$$
\min _{\phi} W(\phi)
$$


For a smooth surface $\Gamma$, the convergence of the Allen-Cahn type phase field Willmore dynamics (3) to the gradient dynamics associated to the Willmore flow is provided in [34] via matched asymptotic expansions. A recent study in [2] provided further development that can handle singular surfaces.

To study the vesicle morphology, it is often additionally required that the vesicle maintains a fixed surface area and a fixed inside volume during the process of shape transformation. In the phase field setting $[14,39]$, the inside volume $\operatorname{Vol}(\Gamma)$ can be approximated by

$$
V(\phi)=\int_{\Omega} \frac{1}{2}(\phi+1) d \mathbf{x}
$$

and the surface area $\operatorname{Surf}(\Gamma)$ by

$$
A(\phi)=\frac{3}{2 \sqrt{2}} \int_{\Omega} \frac{\epsilon}{2}|\nabla \phi|^{2}+\frac{1}{4 \epsilon}\left(\phi^{2}-1\right)^{2} d \mathbf{x} .
$$

By assuming fixed inside volume $\alpha_{0}$ and surface area $\beta_{0}$, we may consider a constrained optimization problem

$$
\min _{\phi} W(\phi), \quad \text { s.t. } \quad V(\phi) \equiv \alpha_{0}, A(\phi) \equiv \beta_{0} .
$$

It was shown in [39] that the tanh profile of the phase field function $\phi$ still can be preserved with the two constraints.

A variety of numerical algorithms have been developed in $[14,16]$ for simulation of dynamics of (4) and (7) by using finite difference discretizations in space and backward or forward Euler schemes in time. Finite element spatial discretizations are studied in [18, 20]. With convergence and error estimates done in $[17,45]$. Most of the existing methods are first-order accurate in time, which is a major limitation. Meanwhile, implicit schemes require solution of large systems while the explicit method developed so far requires very small time step sizes. In this work, we present highorder accurate explicit numerical methods for solving the Allen-Cahn type phase field Willmore dynamics (3) with and without constraints by combining explicit exponential time differencing Runge-Kutta (ETDRK) schemes [6, 19] and spectral spatial discretizations with novel operator splitting stabilization techniques. Such techniques have proven to be effective for other phase field type models $[32,31,30]$. The application to the phase field Willmore dynamics provides new challenges due to the nonlinearities in various terms. Furthermore, the incorporation of surface area and volume constraints into high-order schemes is an issue never discussed in the literature before. To avoid the potential numerical instability due to the use of large penalty parameters in the penalty formulation of the constrained phase field Willmore dynamics problem, we develop a modified augmented Lagrange multiplier (ALM) approach to handle the constraints. Our work here demonstrates that the proposed methods not only have all essential features of explicit time stepping schemes in terms of simple and efficient implementation but also have better stability for large time step sizes and higher order accuracy than previously developed approaches.

The rest of the paper is organized as follows: efficient and stable ETDRK methods for the phased field elastic bending energy model (4) and its dynamics evolution described by (3) are given and discussed in Section 2. In Section 3, we then further develop ETDRK methods that combine the ALM approach with a quasi-Newton updating process to solve the constrained phase field Willmore dynamics, while still keeping good numerical stability and high-order accuracy. Results of numerical benchmark experiments are reported in each of these sections. In Section 4, we provide additional experiments to demonstrate the superiority of the proposed methods in simulating complex morphological membrane patterns. Finally some conclusions are drawn in Section 5 . 


\section{Stabilized ETDRK method for the unconstrained phase field Wilmore dynamics}

We now derive the stabilized ETDRK method for simulating the Allen-Cahn type phase field Willmore dynamics (3). The stabilization is done through operator splitting which can help improving temporal numerical stability without causing a degradation of overall accuracy for large time step size due to the high-order accuracy.

\subsection{Linear splittings for stabilization}

We first adopt an analogous linear splitting scheme used commonly in stabilized numerical methods for the classic Allen-Cahn equation $[19,42,43,31]$ to rewrite $f(\phi)$ as

$$
f(\phi)=\left(\Delta \phi-\frac{\kappa}{\epsilon^{2}} \phi\right)-\frac{1}{\epsilon^{2}}\left(\phi^{3}-(1+\kappa) \phi\right),
$$

where $\kappa$ is a constant splitting parameter for stability and it is often required $\kappa \geq 1$. Then we can transform the equation (3) into

$$
\begin{aligned}
\frac{\partial \phi}{\partial t}= & -\epsilon\left(\Delta^{2} \phi-\frac{\kappa}{\epsilon^{2}} \Delta \phi\right)+\frac{1}{\epsilon} \Delta\left(\phi^{3}-(1+\kappa) \phi\right)+\frac{1}{\epsilon}\left(3 \phi^{2}-1\right)\left(\Delta \phi-\frac{\kappa}{\epsilon^{2}} \phi\right) \\
& -\frac{1}{\epsilon^{3}}\left(3 \phi^{2}-1\right)\left(\phi^{3}-(1+\kappa) \phi\right) \\
= & {\left[-\epsilon\left(\Delta^{2} \phi-\frac{\kappa}{\epsilon^{2}} \Delta \phi\right)+\frac{1}{\epsilon}\left(3 \phi^{2}\right)\left(\Delta \phi-\frac{\kappa}{\epsilon^{2}} \phi\right)\right]+} \\
& {\left[-\frac{1}{\epsilon}\left(\Delta \phi-\frac{\kappa}{\epsilon^{2}} \phi\right)+\frac{1}{\epsilon} \Delta\left(\phi^{3}-(1+\kappa) \phi\right)-\frac{1}{\epsilon^{3}}\left(3 \phi^{2}-1\right)\left(\phi^{3}-(1+\kappa) \phi\right)\right] . }
\end{aligned}
$$

As for the nonlinear term $\frac{1}{\epsilon}\left(3 \phi^{2}\right)\left(\Delta \phi-\frac{\kappa}{\epsilon^{2}} \phi\right)$, since $3 \phi^{2} \geq 0$ we further apply another splitting approach similar to that used in $[5,30]$ for dealing with a variable diffusion mobility and rewrite the term as:

$$
\frac{1}{\epsilon}\left(3 \phi^{2}\right)\left(\Delta \phi-\frac{\kappa}{\epsilon^{2}} \phi\right)=\left[\frac{\mu}{\epsilon}\left(\Delta \phi-\frac{\kappa}{\epsilon^{2}} \phi\right)\right]+\left[\frac{1}{\epsilon}\left(3 \phi^{2}-\mu\right)\left(\Delta \phi-\frac{\kappa}{\epsilon^{2}} \phi\right)\right],
$$

where $\mu>0$ is a new constant parameter. It is required that the first linear term can control the second nonlinear term (i.e., $\mu \geq \max _{|\phi| \leq 1}\left|3 \phi^{2}-\mu\right|$ ) as suggested in [5], which implies

$$
\mu \geq \frac{1}{2}\left(\max _{|\phi| \leq 1} 3 \phi^{2}+\min _{|\phi| \leq 1} 3 \phi^{2}\right)=\frac{3}{2} .
$$

By putting (10) into (9) we finally obtain a stabilized form of the equation (3) as

$$
\begin{aligned}
\frac{\partial \phi}{\partial t}= & {\left[-\epsilon\left(\Delta^{2} \phi-\frac{\kappa}{\epsilon^{2}} \Delta \phi\right)+\frac{\mu}{\epsilon}\left(\Delta \phi-\frac{\kappa}{\epsilon^{2}} \phi\right)\right]+} \\
& {\left[\frac{1}{\epsilon}\left(3 \phi^{2}-\mu-1\right)\left(\Delta \phi-\frac{\kappa}{\epsilon^{2}} \phi\right)+\frac{1}{\epsilon} \Delta\left(\phi^{3}-(1+\kappa) \phi\right)-\frac{1}{\epsilon^{3}}\left(3 \phi^{2}-1\right)\left(\phi^{3}-(1+\kappa) \phi\right)\right] } \\
= & \mathcal{L}(\phi)+\mathcal{N}(\phi),
\end{aligned}
$$

where the linear term

$$
\mathcal{L}(\phi)=-\epsilon \Delta^{2} \phi+\frac{\kappa+\mu}{\epsilon} \Delta \phi-\frac{\mu \kappa}{\epsilon^{3}} \phi
$$


and the nonlinear term

$$
\mathcal{N}(\phi)=\frac{1}{\epsilon}\left(3 \phi^{2}-\mu-1\right)\left(\Delta \phi-\frac{\kappa}{\epsilon^{2}} \phi\right)+\frac{1}{\epsilon} \Delta\left(\phi^{3}-(1+\kappa) \phi\right)-\frac{1}{\epsilon^{3}}\left(3 \phi^{2}-1\right)\left(\phi^{3}-(1+\kappa) \phi\right) .
$$

Remark 1. These two splittings play a crucial role on the stability of the numerical schemes proposed in the paper. The classic splitting schemes with only one parameter $\kappa$ (i.e., $\mu=0$ ) does not work here due to the requirement of (11). A good choice of the parameters $\kappa$ and $\mu$ is to take $\kappa=2$ and $\mu=\frac{3}{2}$, and then (12) can be written as

$$
\begin{aligned}
\frac{\partial \phi}{\partial t}= & {\left[-\epsilon \Delta^{2} \phi+\frac{7}{2 \epsilon} \Delta \phi-\frac{3}{\epsilon^{3}} \phi\right]+} \\
& {\left[\frac{1}{\epsilon}\left(3 \phi^{2}-\frac{5}{2}\right)\left(\Delta \phi-\frac{2}{\epsilon^{2}} \phi\right)+\frac{1}{\epsilon} \Delta\left(\phi^{3}-3 \phi\right)-\frac{1}{\epsilon^{3}}\left(3 \phi^{2}-1\right)\left(\phi^{3}-3 \phi\right)\right] . }
\end{aligned}
$$

We take this set of parameters in all numerical experiments.

\subsection{Spectral spatial discretization under periodic boundary condition}

Let us consider a regular domain in $\mathbb{R}^{3}, \Omega=\left\{x_{b}<x<x_{e}, y_{b}<y<y_{e}, z_{b}<z<z_{e}\right\}$, and a periodical boundary condition is imposed for the problem. Let us discretize the spatial domain $\Omega$ by a rectangular mesh which is uniform in each direction as follows:

$$
\left(x_{i}, y_{j}, z_{k}\right)=\left(x_{b}+i h_{x}, y_{b}+j h_{y}, z_{b}+k h_{z}\right)
$$

for $0 \leq i \leq N_{x}, 0 \leq j \leq N_{y}$ and $0 \leq k \leq N_{z}$. The mesh size in each dimension is then given by $h_{x}=\left(x_{e}-x_{b}\right) / N_{x}, h_{y}=\left(y_{e}-y_{b}\right) / N_{y}$ and $h_{z}=\left(z_{e}-z_{b}\right) / N_{z}$, respectively. Let $\phi_{i, j, k}=\phi_{i, j, k}(t) \approx$ $u\left(t, x_{i}, y_{j}, z_{k}\right)$ for $0 \leq i \leq N_{x}, 0 \leq j \leq N_{y}$ and $0 \leq k \leq N_{z}$ denote the approximate solution. Then the set of unknowns are

$$
\boldsymbol{\Phi}=\left(\phi_{i, j, k}\right)_{0: N_{x}-1,0: N_{y}-1,0: N_{z}-1} .
$$

The Laplacian operator in the spectral space is corresponding to the following spectrum

$$
\lambda_{i, j, k}=-\lambda_{x}^{2}(i)-\lambda_{y}^{2}(j)-\lambda_{z}^{2}(k)
$$

with

$$
\begin{aligned}
& \lambda_{x}(i)= \begin{cases}\frac{2 \pi}{x_{e}-x_{b}} i, & 0 \leq i \leq N_{x} / 2, \\
\frac{2 \pi}{x_{e}-x_{b}}\left(N_{x}-i\right), & N_{x} / 2<i \leq N_{x}-1,\end{cases} \\
& \lambda_{y}(j)= \begin{cases}\frac{2 \pi}{y_{e}-y_{b}} j, & 0 \leq j \leq N_{y} / 2, \\
\frac{2 \pi}{y_{e}-y_{b}}\left(N_{y}-j\right), & N_{y} / 2<j \leq N_{y}-1,\end{cases} \\
& \lambda_{z}(k)= \begin{cases}\frac{2 \pi}{z_{e}-z_{b}} k, & 0 \leq k \leq N_{z} / 2, \\
\frac{2 \pi}{z_{e}-z_{b}}\left(N_{z}-k\right), & N_{z} / 2<k \leq N_{z}-1 .\end{cases}
\end{aligned}
$$

Let $\odot$ denote the element-wise multiplication of two matrices. By taking fast Fourier transform (FFT) [33] on both sides of the equation (12), we get

$$
\mathbf{U}_{t}=\mathbf{L} \odot \mathbf{U}+\mathbf{N}(\mathbf{U}),
$$


where $\mathbf{U}=\operatorname{FFT}(\mathbf{\Phi})=\left(u_{i, j, k}\right)_{0: N_{x}-1,0: N_{y}-1,0: N_{z}-1}$, the transformed linear term from (13) is

$$
\mathbf{L} \odot \mathbf{U}=\left(\ell_{i, j, k} u_{i, j, k}\right), \quad \ell_{i, j, k}=-\epsilon \lambda_{i, j, k}^{2}+\frac{\kappa+\mu}{\epsilon} \lambda_{i, j, k}-\frac{\kappa \mu}{\epsilon^{3}},
$$

and the transformed nonlinear term from (14) is

$$
\mathbf{N}(\mathbf{U})=\operatorname{FFT}(\mathcal{N}(\operatorname{iFFT}(\mathbf{U}))) .
$$

Since $\lambda_{i, j, k} \leq 0$, it is obvious that $\ell_{i, j, k}<0$ if $\kappa, \mu>0$. More specifically, we could write (17) point-wisely as

$$
\left(u_{i, j, k}\right)_{t}=\ell_{i, j, k} u_{i, j, k}+[\mathbf{N}(\mathbf{U})]_{i, j, k},
$$

for $0 \leq i \leq N_{x}-1,0 \leq j \leq N_{y}-1$, and $0 \leq k \leq N_{z}-1$. In the following we will develop high-order Runge-Kutta approximations based on exponential time differencing for the time integration of (18).

Remark 2. The classic semi-implicit Euler (single-step) scheme could be used to solve the stabilized equation (18): in a single time step from $t_{n}$ to $t_{n+1}=t_{n}+\Delta t_{n}$, find $u_{i, j, k}\left(t_{n+1}\right)$ from $\left(u_{i, j, k}\left(t_{n+1}\right)-\right.$ $\left.u_{i, j, k}\left(t_{n}\right)\right) / \Delta t_{n}=\ell_{i, j, k} u_{i, j, k}\left(t_{n+1}\right)+\left[\mathbf{N}\left(\mathbf{U}^{n}\right)\right]_{i, j, k}$. However, such scheme is only of first order accurate in time and the splitting error can have a dominating effect and thus not efficient for accurately simulating the dynamics over the whole long evolution time. The high order schemes developed in this work can significantly improve the accuracy by reducing the error of time-stepping and the effect of the splitting error due to stabilization.

\subsection{Exponential time differencing Runge-Kutta approximations}

The exponential time differencing (ETD) method [6,32] is used for explicitly and accurately solving the semi-discrete system (17). Let $\Delta t_{n}$ denote the time step size at time $t_{n}$. Integrating the equation (18) over a single time step from $t_{n}$ to $t_{n+1}=t_{n}+\Delta t_{n}$, we obtain

$$
u_{i, j, k}\left(t_{n+1}\right)=e^{\ell_{i, j, k} \Delta t_{n}} u_{i, j, k}\left(t_{n}\right)+e^{\ell_{i, j, k} \Delta t_{n}} \int_{0}^{\Delta t_{n}} e^{-\ell_{i, j, k} \tau}\left[\mathbf{N}\left(\mathbf{U}\left(t_{n}+\tau\right)\right)\right]_{i, j, k} d \tau .
$$

The equation (19) is exact, and various ETD based methods can be used: first approximate the nonlinear part $[\mathbf{N}(\mathbf{U})]_{i, j, k}$ using polynomial interpolations and then perform exact integrations on the produced new integrands $[6,31]$.

Denote the approximation to $\mathbf{U}\left(t_{n}\right)=\left(u_{i, j, k}\left(t_{n}\right)\right)$ by $\mathbf{U}^{n}=\left(u_{i, j, k}^{n}\right)$, then the first-order scheme by the ETD Euler approximation, ETD1 (or ETD1RK), is given by

$$
\mathbf{U}^{n+1}=\operatorname{ETD} 1 R K\left(\mathbf{U}^{n}, \Delta t_{n}, \mathcal{L}, \mathcal{N}\right): \quad u_{i, j, k}^{n+1}=e^{\ell_{i, j, k} \Delta t_{n}} u_{i, j, k}^{n}+\ell_{i, j, k}^{-1}\left(e^{\ell_{i, j, k} \Delta t_{n}}-1\right)\left[\mathbf{N}\left(\mathbf{U}^{n}\right)\right]_{i, j, k} .
$$

Higher order ETD schemes can be further derived based on multi-step or Runge-Kutta approximations. For instance, the second, third and fourth order ETD Runge-Kutta schemes (which we refer as ETD2RK, ETD3RK and ETD4RK respectively) can be found in [6]. For the equation (17) we list below the formulae for the second-order scheme (ETD2RK)

$$
\begin{aligned}
& \mathbf{U}^{n+1}=\operatorname{ETD} 2 \operatorname{RK}\left(\mathbf{U}^{n}, \Delta t_{n}, \mathcal{L}, \mathcal{N}\right): \\
& \left\{\begin{array}{l}
\mathbf{A}=\left(a_{i, j, k}\right)=\operatorname{ETD} 1 \mathrm{RK}\left(\mathbf{U}^{n}, \Delta t_{n}, \mathcal{L}, \mathcal{N}\right), \\
u_{i, j, k}^{n+1}=a_{i, j, k}+\Delta t_{n}^{-1} \ell_{i, j, k}^{-2}\left(e^{\ell_{i, j, k} \Delta t_{n}}-1-\ell_{i, j, k} \Delta t_{n}\right)\left[\mathbf{N}(\mathbf{A})-\mathbf{N}\left(\mathbf{U}^{n}\right)\right]_{i, j, k},
\end{array}\right.
\end{aligned}
$$


and the fourth-order scheme (ETK4RK)

$$
\begin{aligned}
& \mathbf{U}^{n+1}=\operatorname{ETD} 4 \mathrm{RK}\left(\mathbf{U}^{n}, \Delta t_{n}, \mathcal{L}, \mathcal{N}\right):
\end{aligned}
$$

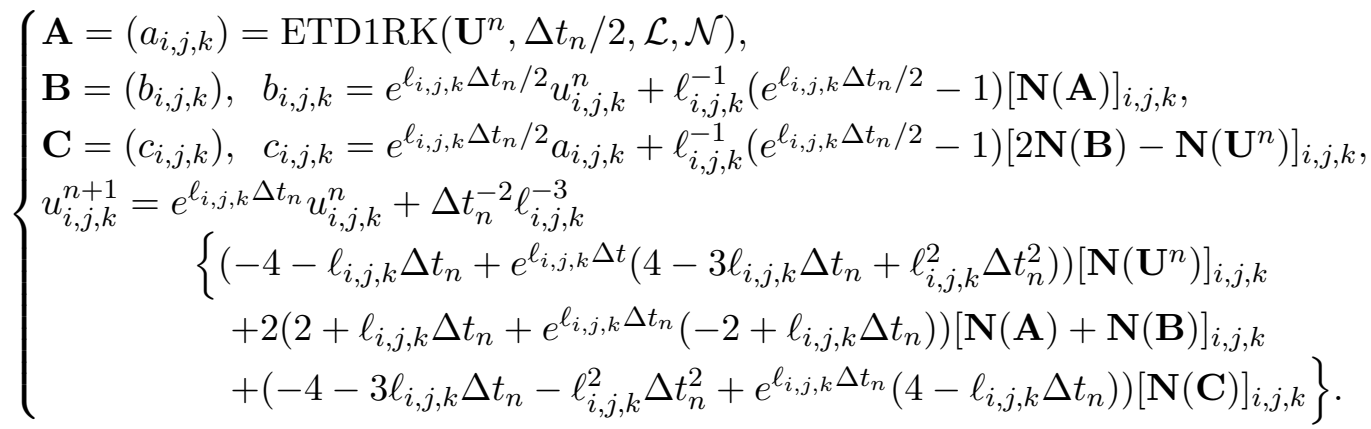

The stabilized ERDTK method then can be presented as follows:

Algorithm 1 (Stabilized ETDRK method for unconstrained phase field Willmore dynamics). Input: the rectangular domain $\Omega \in \mathbb{R}^{3}$, a spatial grid with sizes $h_{x}, h_{y}$ and $h_{z}$ respectively in each dimension, the intial configuration $\boldsymbol{\Phi}_{0}$, the end time $T>0$, the accuracy order $q$, and the time step sizes $\left\{\Delta t_{n}\right\}_{n=0}^{N_{t}-1}$ such that $\sum_{n=0}^{N_{t}-1}=T$.

1. $\mathbf{U}^{0}=\operatorname{FFT}\left(\boldsymbol{\Phi}^{0}\right)$;

2. for $n=0,1, \cdots, N_{t}-1$ do

Compute $\mathbf{U}^{n+1}=\operatorname{ETD} q \operatorname{RK}\left(\mathbf{U}^{n}, \Delta t_{n}, \mathcal{L}, \mathcal{N}\right) ;$

end

3. $\boldsymbol{\Phi}^{N_{t}}=\mathrm{iFFT}\left(\mathbf{U}^{N_{t}}\right)$.

It is easy to check it costs totally $q$ three-dimensional FFTs and $q$ iFFTs per time step for the $q$-th order ETDRK scheme due to calculations of $\mathbf{N}(\cdot)$.

\subsection{Tests of unconstrained phase field Willmore dynamics}

We now verify the accuracy and stability of the proposed method through numerical examples. The experiments were run on a desktop with $2.8 \mathrm{GHz}$ Intel Xeon CPU and 12GB memory. The computation domain was set to be $[-\pi, \pi]^{3}$ for all experiments.

\subsubsection{Experiment with two merging spheres}

Let us consider the phase field Willmore dynamics starting with two close-by spheres. We chose the uniform spatial mesh $128^{3}$ (i.e., the mesh size $h=2 \pi / 128$ ) and set $\epsilon=3 h$. The initial phase field function was chosen as

$$
\begin{aligned}
\phi(x, y, z)= & \tanh \left(\frac{0.28 \pi-\sqrt{\left(x^{2}+y^{2}+(z+0.35 \pi)^{2}\right.}}{\sqrt{2} \epsilon}\right) \\
& +\tanh \left(\frac{0.28 \pi-\sqrt{\left(x^{2}+y^{2}+(z-0.35 \pi)^{2}\right.}}{\sqrt{2} \epsilon}\right)+1.0,
\end{aligned}
$$

see Figure 1-(a) for an illustration. We tested Algorithm 1 of first-order (ETD1RK), second-order (ETD2RK) and fourth-order (ETD4RK) with time step sizes $\Delta t=10^{-2}, 10^{-3}, 10^{-4}$ respectively. 
The simulation time was from $t=0$ to $t=10.0$ and it was observed that from time $t=4.0$ to 10.0 the solution remained essentially unchanged.

Figure 1 shows the typical merging transformation process of the two close-by spheres described in (23) without constraints, obtained by the stabilized ETD4RK scheme with the time step size $\Delta t=10^{-3}$ : the two spheres first merge together and gradually change to a larger sphere. This is a common behavior observed in three-dimensional phase field or level set based Willmore flow models, see $[15,8,21]$ and references cited therein. Note that Figures 1 -(b) to (h) are cut views with a $90^{\circ}$ cutting angle so as to better illustrate the three dimensional membrane structure. Such cut view shots are also used later when presenting results of other experiments. Figure 2 presents plots of the energy evolution for these tests. The first row compares the energy curves under different time step sizes for each of the three stabilized ETDRK schemes, while the second row reorganizes the curves using different schemes but the same time step size. It is easy to see that all schemes worked stably with all time step sizes, and converged as the time step size decreased. Figure 2-(f) reveals that for $\Delta t=10^{-4}$, the energy curves based on different schemes are nearly indistinguishable. To achieve the same effect with larger step sizes, one can find a good match between the curves $\Delta t=10^{-3}$ and $\Delta t=10^{-4}$ for the ETD4RK scheme in Figure 2-(c), but not for other choices of schemes or step sizes.

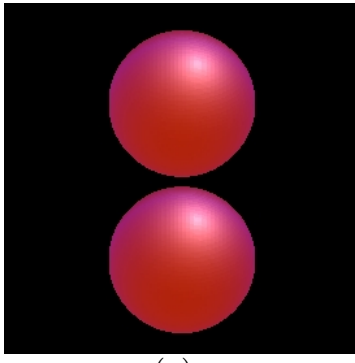

(a)

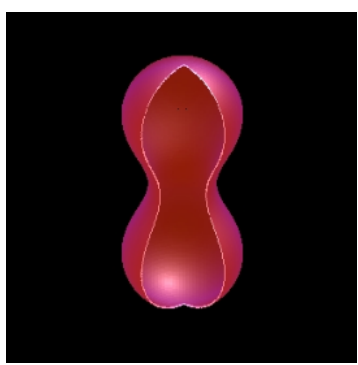

(e)

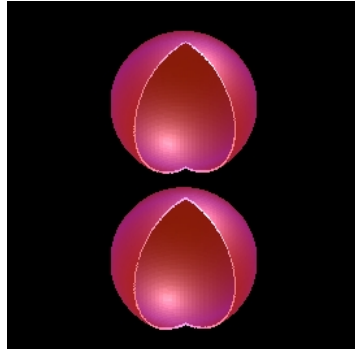

(b)

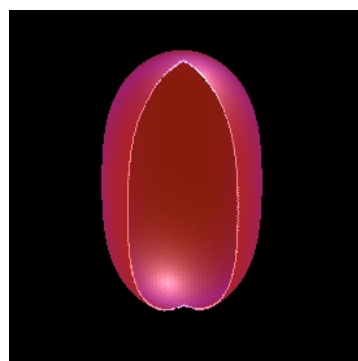

(f)

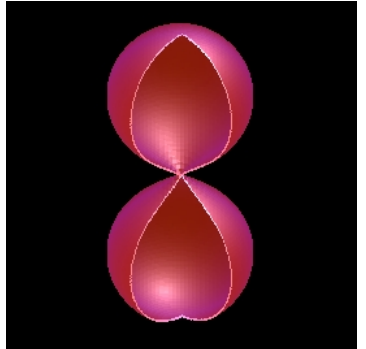

(c)

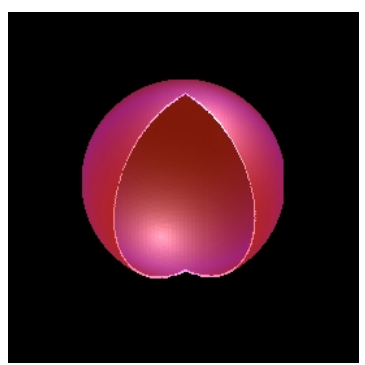

(g)

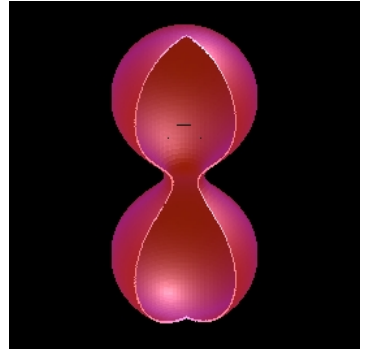

(d)

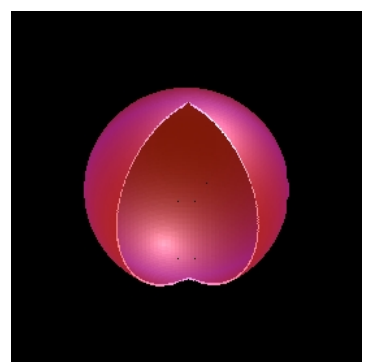

(h)

Figure 1: Simulation of the unconstrained phase field Willmore dynamics of two close-by spheres (23) using the stabilized ETD4RK scheme with $\Delta t=10^{-3}$. (a) the initial shape $(t=0)$; (b) the initial shape in cut view; from (b) to $(\mathrm{h})$ : the transformed shape in cut view under the unconstrained phase field Willmore dynamics at time $t=0,0.018,0.03,0.1,1.0,4.0,10.0$, respectively.

To further test the convergence rates of these stabilized ETDRK schemes, we performed simulations for a smaller time interval $[0,0.1]$, during such time, the two spheres undergo the merging process as seen from Figure 1-(b) to Figure 1-(e)). We use the solution produced by the ETD4RK scheme with $\Delta t=10^{-5}$ as the approximate exact solution (benchmark) and then compute the errors in energy for all schemes with larger step sizes. The benchmark energy in the error calcu- 


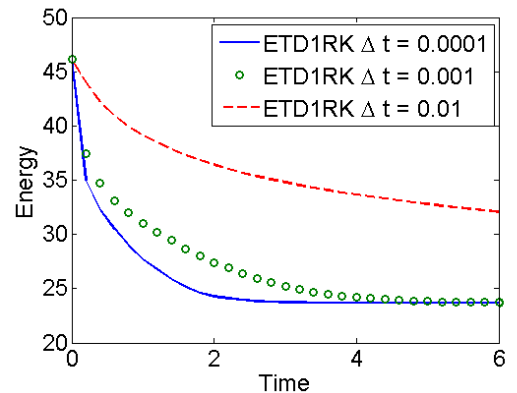

(a)

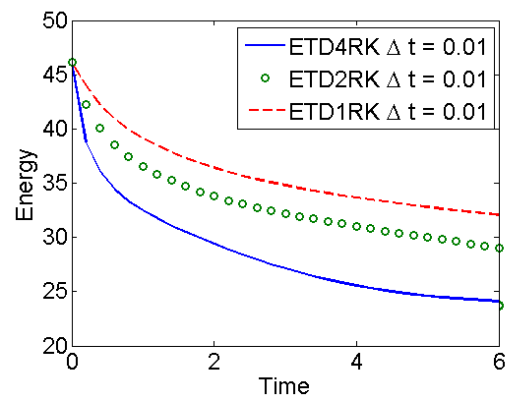

(d)

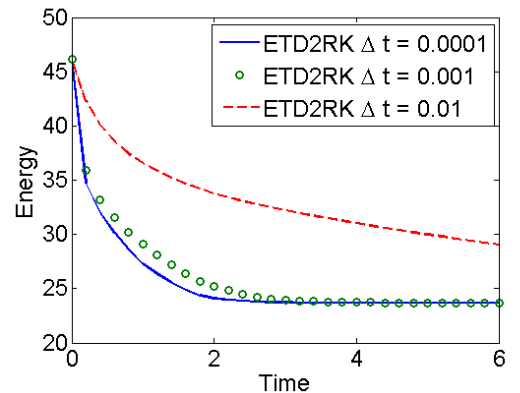

(b)

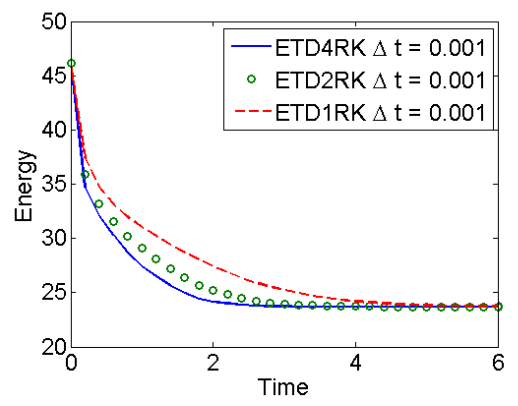

(e)

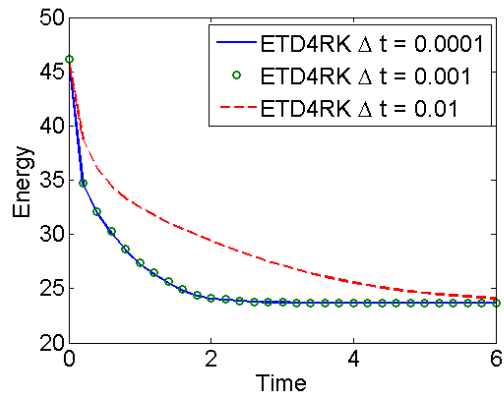

(c)

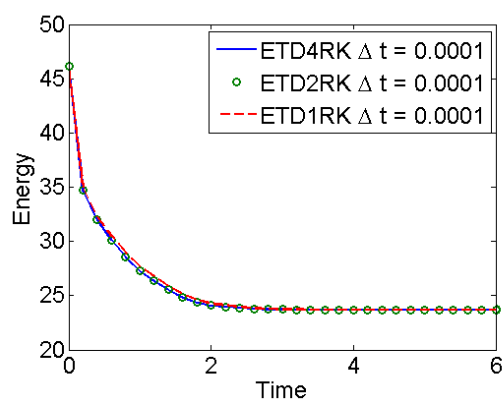

(f)

Figure 2: Plots of energy evolution for the unconstrained phase field Willmore dynamics of two close-by spheres (23) obtained by the stabilized ETD1RK, ETD2RK and ETD4RK schemes with different time step sizes $\Delta t=$ $0.01,0.001,0.0001$, respectively.

lation is 37.4426720750. Table 1 presents the energies, errors and corresponding convergence rates at the time based on the data at $t=0.1$ for all schemes with time step sizes being halved from $\Delta t=4 \times 10^{-3}$ to $3.125 \times 10^{-5}$. These data are also used to produce Figure 3 which shows energy errors against time step sizes in a logarithmic scale for the ETD1RK, ETD2RK and ETD4RK schemes. We can see from both the curves and the table that the numerically computed convergence rates all gradually approach to the theoretical values. Moreover, to get an energy error comparable to that of ETD1RK with $\Delta t=3.125 \times 10^{-5}$, one can enlarge the step size about 4 to 8 times to $1.25 \times 10^{-4}$ or $2.5 \times 10^{-4}$ for ETD2RK, but for ETD4RK, the step size can go to $\Delta t=10^{-3}$ which is 32 times larger than ETD1RK. Since the ETD4RK scheme carries a computational cost about 4 times of the ETD1RK scheme per step, we see that the high-order scheme ETD4RK basically provides a factor of 8 speed up at this particular accuracy level for this special test case.

Remark 3. In the literature there are some discussions about the experiment of merging of two close-by spheres [21]. A single sphere itself is a Willmore energy minimizer. Two separated spheres in the perfect sharp interface case will remain separated as an equilibrium state. In phase field modeling, due to diffuse interface approximation, two spheres can "sense" each other and thus merge with each other. The effect of "sensing" of two spheres decreases exponentially when we decrease the transition layer width or increase the distance between them. Some modified phase field Willmore dynamics formulations [21] are able to prevent the merging of two disks in two dimensions, but not that of two spheres in three dimensions. 


\begin{tabular}{|c||c|c|c||c|c|c||c|c|c|}
\hline \multicolumn{1}{|c||}{} & \multicolumn{3}{c||}{ ETD4RK } & \multicolumn{3}{c||}{ ETD2RK } & \multicolumn{3}{c|}{ ETD1RK } \\
\hline$\Delta t$ & Energy & Error & Rate & Energy & Error & Rate & Energy & Error & Rate \\
\hline \hline $4.000 \times 10^{-3}$ & 38.81564 & 1.3729679 & - & 41.78258 & 4.3399034 & - & 43.47617 & 6.0335012 & - \\
\hline $2.000 \times 10^{-3}$ & 37.85254 & 0.4098679 & 1.74 & 39.94112 & 2.4984474 & 0.80 & 41.68623 & 4.2435562 & 0.51 \\
\hline $1.000 \times 10^{-3}$ & 37.53338 & 0.0907079 & 2.18 & 38.59035 & 1.1476814 & 1.12 & 40.03877 & 2.5960990 & 0.71 \\
\hline $5.000 \times 10^{-4}$ & 37.45921 & 0.0165414 & 2.46 & 37.87870 & 0.4360310 & 1.40 & 38.87828 & 1.4356116 & 0.86 \\
\hline $2.500 \times 10^{-4}$ & 37.44525 & 0.0025780 & 2.68 & 37.58700 & 0.1443304 & 1.60 & 38.19089 & 0.7482149 & 0.94 \\
\hline $1.250 \times 10^{-4}$ & 37.44301 & 0.0003385 & 2.93 & 37.48619 & 0.0435143 & 1.73 & 37.82232 & 0.3796513 & 0.98 \\
\hline $6.250 \times 10^{-5}$ & 37.44271 & 0.0000370 & 3.19 & 37.45498 & 0.0123035 & 1.82 & 37.63343 & 0.1907555 & 0.99 \\
\hline $3.125 \times 10^{-5}$ & 37.44268 & 0.0000032 & 3.52 & 37.44600 & 0.0033254 & 1.90 & 37.53821 & 0.0955397 & 1.00 \\
\hline
\end{tabular}

Table 1: The energies, errors and corresponding convergence rates at time $t=0.1$ by the stabilized ETD1RK, ETD2RK and ETD4RK schemes for the unconstrained phase field Willmore dynamics of two close-by spheres (23).

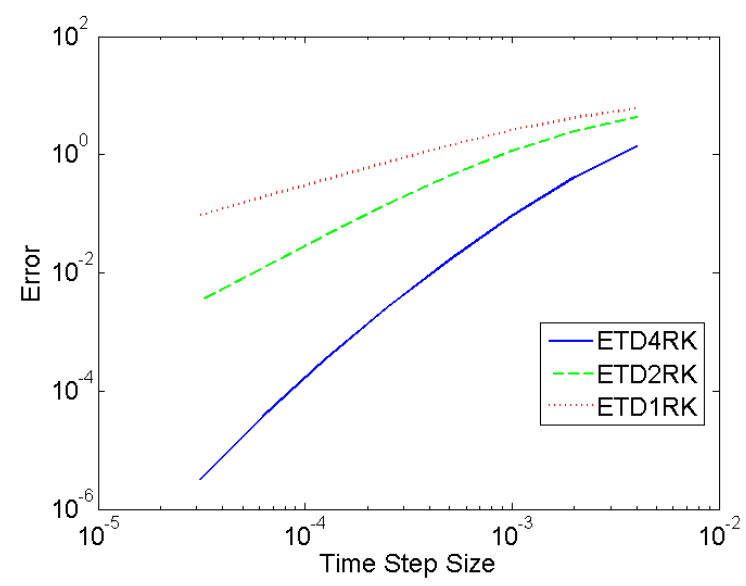

Figure 3: Logarithmic plots of energy errors vs. time step sizes of the stabilized ETD1RK, ETD2RK and ETD4RK schemes for the unconstrained phase field Willmore dynamics of two close-by spheres (23).

\subsubsection{Experiment with an expanding cylinder}

Our next experiment is to choose the initial phase field function as

$$
\phi(x, y, z)=\tanh \left(\frac{0.1 \pi-\sqrt{x^{2}+y^{2}}}{\sqrt{2} \epsilon}\right),
$$

which describes a cylinder with a radius $0.1 \pi$. We again used the same uniform spatial mesh $128^{3}$ and set $\epsilon=3 h$. Following the phase field Willmore dynamics, the horizontal radius of the cylinder will gradually expand with a slowing down speed [8] as shown in Figure 4. This example is an analog to the expansion of a circle in the two-dimensional space by adding a perpendicular coordinate $z$. Table 2 presents the energies, errors and corresponding convergence rates at the time $t=100.0$ based on the data for all the schemes with time step sizes being halved from $\Delta t=4 \times 10^{-3}$ to $3.125 \times 10^{-5}$. The benchmark energy for the error calculations is 9.4941634163 obtained by using the ETD4RK scheme with $\Delta t=10^{-5}$. We can see from Table 2 that the convergence rates of ETD1RK and ETD2RK gradually reach or get quite close their respective optimal values 1.0 and 2.0 along the refinement of time step sizes. Those for the high-order scheme ETD4RK are similarly improving but still somehow lower than its respective theoretical optimal value at the last level. 


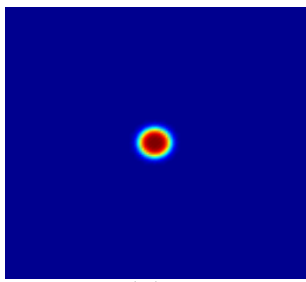

(a)

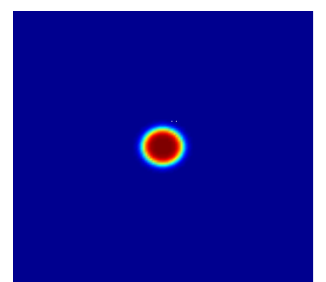

(b)

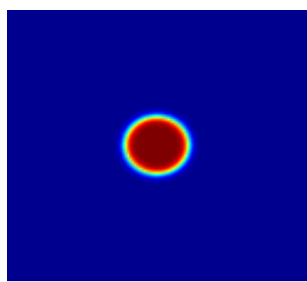

(c)

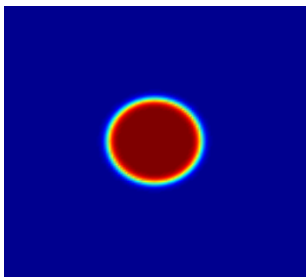

(d)

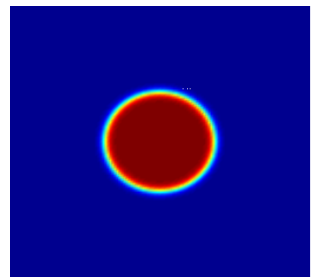

(e)

Figure 4: Plots of the cross section (top views) for the unconstrained phase field Willmore dynamics of a cylinder (24) obtained by the stabilized ETD4RK schemes at time $t=0,1.0,10.0,50.0,100.0$, respectively.

\begin{tabular}{|c||r|r|r||r|r|r||r|r|r|}
\hline \multicolumn{1}{|c||}{} & \multicolumn{3}{c||}{ ETD4RK } & \multicolumn{3}{|c||}{ ETD2RK } & \multicolumn{3}{|c|}{ ETD1RK } \\
\hline$\Delta t$ & Energy & Error & Rate & Energy & Error & Rate & Energy & Error & Rate \\
\hline \hline $4.000 \times 10^{-3}$ & 16.19369 & 6.6995310 & - & 21.12993 & 11.6357625 & - & 24.94573 & 15.4515709 & - \\
\hline $2.000 \times 10^{-3}$ & 13.73555 & 4.2413877 & 0.66 & 17.86023 & 8.3660620 & 0.48 & 21.07735 & 11.5831873 & 0.42 \\
\hline $1.000 \times 10^{-3}$ & 11.76218 & 2.2680195 & 0.90 & 15.12206 & 5.6278974 & 0.57 & 17.77101 & 8.2768456 & 0.48 \\
\hline $5.000 \times 10^{-4}$ & 10.42229 & 0.9281219 & 1.29 & 12.88801 & 3.3938507 & 0.73 & 14.99473 & 5.5005694 & 0.59 \\
\hline $2.500 \times 10^{-4}$ & 9.77746 & 0.2832989 & 1.71 & 11.24557 & 1.7514055 & 0.95 & 12.81977 & 3.3256092 & 0.73 \\
\hline $1.250 \times 10^{-4}$ & 9.56182 & 0.0676601 & 2.07 & 10.25449 & 0.7603277 & 1.20 & 11.32810 & 1.8339380 & 0.86 \\
\hline $6.250 \times 10^{-5}$ & 9.50688 & 0.0127156 & 2.41 & 9.75817 & 0.2640031 & 1.53 & 10.44071 & 0.9465482 & 0.95 \\
\hline $3.125 \times 10^{-5}$ & 9.49597 & 0.0018088 & 2.81 & 9.56862 & 0.0744571 & 1.82 & 9.96766 & 0.4734986 & 1.00 \\
\hline
\end{tabular}

Table 2: The energies, errors and corresponding convergence rates at time $t=100.0$ by the stabilized ETD1RK, ETD2RK and ETD4RK schemes for the unconstrained phase field Willmore dynamics of an expanding cylinder (24).

\subsubsection{Significant performance enhancement over old methods}

The stabilized high-order ETDRK schemes can offer a huge performance improvement over the old methods such as the widely used forward-Euler method [15] which has to use very small time step sizes in order to avoid numerical instability. We compare the stabilized ETD4RK scheme with the forward-Euler method using the experiment of two close-by spheres. We set $\epsilon=3 h$ for both methods and the simulation ending time was chosen to be $t=1.0$. The mesh of $64^{3}$ is first used. Note that the forward-Euler method is also allowed to automatically adjust its time step size at each step to ensure the energy decreasing as done in [15], and we find numerically that the time step size actually is always kept around $10^{-6}$. We then run the ETD4RK scheme with a fixed time step size $10^{-3}$. The produced energy curves are plotted in Figure 5 and one can see both methods give almost the identical energy evolution. Yet the forward-Euler scheme takes 42815 seconds to arrive at the time $t=1.0$ but only 262 seconds for the ETD4RK scheme (about 163 times faster). For the finer spatial mesh of $128^{3}$, we find that the forward-Euler method needs a smaller time step size of around $10^{-7}$ to get stable energy decreasing in this case, and the ETD4RK scheme could obtain similar accuracy with a time step size of $5 \times 10^{-4}$. The running time used by the ETD4RK scheme is more than 800 times less than that by the forward-Euler method. Thus the efficiency of the stabilized high-order ETD4RK scheme is very evident. Such huge performance improvement would enable us to practically carry out with fine meshes numerical simulations for more complicated experiments as those presented in $[25,26]$, which are under our investigation.

Parallelization can also help significantly improve the performance of the ETDRK method. When using the ETD4RK scheme with $\Delta t=10^{-3}$, we find that our sequential code takes an average of 2.82 seconds per time step with the $128^{3}$ mesh for this example and 0.26 seconds with 


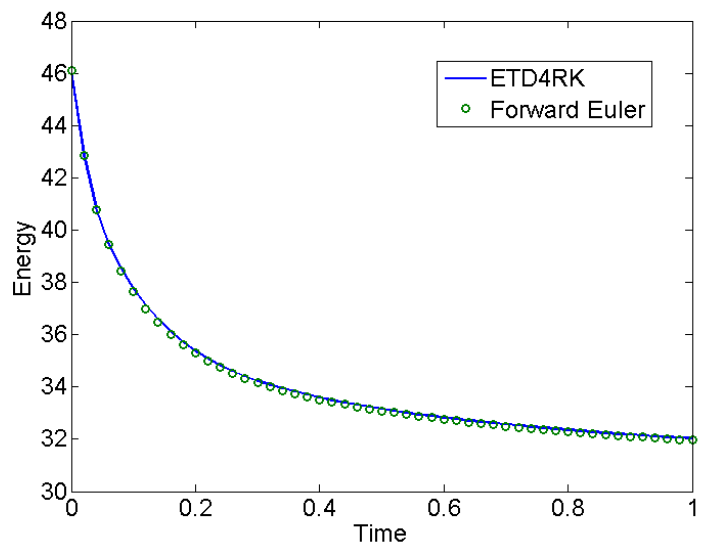

Figure 5: Plots of energy evolution simulated by the ETD4RK scheme and the forward-Euler method with the $64^{3}$ mesh.

the $64^{3}$ mesh. With a simple OpenMP parallelization of the FFT using 8 cores, the average running time per time step is decreased to only 0.55 seconds and 0.053 seconds for the $128^{3}$ and $64^{3}$ meshes respectively. Thus, we get a speedup of roughly 5 folds in performance over the sequential case.

\section{Stabilized ETDRK method with modified ALM for the constrained phase field Willmore dynamics}

For the phase field model with constraints, i.e., the constrained phase field Willmore dynamics problem, we will make use of the Lagrange multiplier approach. As described in [16], the gradient flow can be written as

$$
\begin{aligned}
\phi_{t} & =-\frac{\delta W(\phi)}{\delta \phi}-\lambda_{1}(t) \frac{\delta V(\phi)}{\delta \phi}-\lambda_{2}(t) \frac{\delta A(\phi)}{\delta \phi} \\
& =-\frac{\delta W(\phi)}{\delta \phi}-\frac{\lambda_{1}(t)}{2}+\frac{3 \epsilon \lambda_{2}(t)}{2 \sqrt{2}} f(\phi)
\end{aligned}
$$

where $\lambda_{1}(t)$ and $\lambda_{2}(t)$ are some appropriate time-dependent Lagrange multipliers such that the constraints $V(\phi) \equiv \alpha_{0}$ and $A(\phi) \equiv \beta_{0}$ always hold. In [16], a set of equations were introduced to calculate the two Lagrange multipliers directly with $\phi$ at each time step, however, the two constraints can only be preserved at no more than first-order accuracy in such a method. In addition, a predictor-corrector approach was used to iteratively find $\lambda_{1}(t)$ and $\lambda_{2}(t)$ in practice, and the time step size still needs to be taken very small to avoid numerical instabilities. In this section we propose a modified augmented Lagrange multiplier approach to couple with the stabilized ETDRK schemes to iteratively solve $\lambda_{1}(t)$ and $\lambda_{2}(t)$ and provides significant improvement in accuracy and stability.

\subsection{Penalty formulation}

To motivate our new approach, we begin with a penalty formulation. A simple alternate approach to approximate (25) is to use the penalty formulation as done in [16], i.e., choose two 
large positive constants $M_{1}$ and $M_{2}$ to penalize the constraints and then solve the the following unconstrained minimization problem

$$
\min _{\phi} W_{M}(\phi)=W(\phi)+\frac{1}{2} M_{1}\left(V(\phi)-\alpha_{0}\right)^{2}+\frac{1}{2} M_{2}\left(A(\phi)-\beta_{0}\right)^{2} .
$$

The corresponding Allen-Cahn type dynamics becomes

$$
\begin{aligned}
\phi_{t} & =-\frac{\delta W_{M}(\phi)}{\delta \phi} \\
& =-\epsilon \Delta f+\frac{1}{\epsilon}\left(3 \phi^{2}-1\right) f-\frac{M_{1}}{2}\left(V(\phi)-\alpha_{0}\right)+\frac{3 \epsilon M_{2}}{2 \sqrt{2}}\left(A(\phi)-\beta_{0}\right) f(\phi) .
\end{aligned}
$$

The above equation (27) may be seen as an approximation of the Lagrange multiplier formulation (25) with

$$
\lambda_{1}(t) \approx M_{1}\left(V(\phi)-\alpha_{0}\right), \quad \lambda_{2}(t) \approx M_{2}\left(A(\phi)-\beta_{0}\right) .
$$

The formulations given by (25) and (28) become equivalent only when $M_{1}, M_{2} \rightarrow \infty$.

Applying the splitting schemes presented in the preceding section, we rewrite the dynamic equation as $\phi_{t}=\mathcal{L}(\phi)+\mathcal{N}_{M}(\phi)$ where the linear term $\mathcal{L}(\phi)$ is identical to (13) and the nonlinear term is now

$$
\mathcal{N}_{M}(\phi)=\mathcal{N}(\phi)-\frac{M_{1}}{2}\left(V(\phi)-\alpha_{0}\right)+\frac{3 \epsilon M_{2}}{2 \sqrt{2}}\left(A(\phi)-\beta_{0}\right) f(\phi) .
$$

The additional terms in $\mathcal{N}_{\mathcal{M}}(\phi)$ bring strong stiffness to the system when $M_{1}$ and $M_{2}$ are very large in order to assure accuracy of the constraints.

\subsection{Modified augmented Lagrange multiplier approach}

To overcome the difficulty produced by the penalty formation, let us turn to the augmented Lagrange multiplier (ALM) method for solving the constrained problem. The classic version of the ALM method was first discussed in [28], in which the basic idea is to repeatedly solve the following unconstrained minimization objective

$$
\min _{\phi} W_{A M}(\phi)=W(\phi)+\frac{1}{2} M_{1}\left(V(\phi)-\alpha_{0}\right)^{2}+\frac{1}{2} M_{2}\left(A(\phi)-\beta_{0}\right)^{2}+\gamma_{1}\left(V(\phi)-\alpha_{0}\right)+\gamma_{2}\left(A(\phi)-\beta_{0}\right)
$$

where $\gamma_{1}$ and $\gamma_{2}$ are adjusted at each iteration by

$$
\gamma_{1} \leftarrow \gamma_{1}+M_{1}\left(V(\phi)-\alpha_{0}\right), \quad \gamma_{2} \leftarrow \gamma_{2}+M_{2}\left(A(\phi)-\beta_{0}\right) .
$$

Note $M_{1}, M_{2}$ can be some fixed constants at all iterations in such approach. As the number of iterations goes to infinity, $V(\phi) \rightarrow \alpha_{0}, A(\phi) \rightarrow \beta_{0}$, and (30) converges to the corresponding Lagrange multipliers formulation. The main purpose of this approach is to use small $M_{1}$ and $M_{2}$ together with the adjustment of $\gamma_{1}$ and $\gamma_{2}$ to preserve the volume and surface area and at the same time improve the stability of the system compared with the penalty formulation.

The gradient flow corresponding to the dynamics of the constrained model (30) can be written in the following way as

$$
\begin{aligned}
\phi_{t} & =-\frac{\delta W(\phi)}{\delta \phi}-M_{1}\left(V(\phi)-\alpha_{0}+\gamma_{1}(t)\right) \frac{\delta V(\phi)}{\delta \phi}-M_{2}\left(A(\phi)-\beta_{0}+\gamma_{2}(t)\right) \frac{\delta A(\phi)}{\delta \phi} \\
& =-\frac{\delta W(\phi)}{\delta \phi}-M_{1}(V(\phi)-\alpha(t))+\frac{3 \epsilon M_{2}}{2 \sqrt{2}}(A(\phi)-\beta(t)) f(\phi),
\end{aligned}
$$


where $\alpha(t)=\alpha_{0}-\gamma_{1}(t)$ and $\beta(t)=\beta_{0}-\gamma_{2}(t)$ are two time-dependent variables used to make the volume and surface area constraints satisfied.

Compared with the gradient flow of the classic Lagrange multiplier formulation (25) for the phase field model with constraints, it is easy to see that

$$
\begin{aligned}
& \lambda_{1}(t)=M_{1}(V(\phi)-\alpha(t))=M_{1}\left(\alpha_{0}-\alpha(t)\right), \\
& \lambda_{2}(t)=M_{2}(A(\phi)-\beta(t))=M_{2}\left(\beta_{0}-\beta(t)\right) .
\end{aligned}
$$

Thus, we are solving a problem equivalent to (25) according to (33). Furthermore, $\alpha(t)$ and $\beta(t)$ in (32) are determined by making $V(\phi) \equiv \alpha_{0}$ and $A(\phi) \equiv \beta_{0}$ hold.

Consider the time discretization, suppose at the time $t_{n}$, the solution $\phi^{n}$ of the gradient flow (32) is found and satisfies the volume and surface area constraints. With $\phi^{n}$ at time $t_{n}$, we solve $\phi^{n+1}$ from the problem

$$
\phi_{t}=\mathcal{L}(\phi)+\mathcal{N}_{A M}(\phi), \quad t \in\left[t_{n}, t_{n+1}\right], \quad \text { with }\left.\phi\right|_{t=t_{n}}=\phi^{n}
$$

where the linear term $\mathcal{L}(\phi)$ is again the same as (13) and the nonlinear term is

$$
\mathcal{N}_{A M}(\phi, \tilde{\alpha}, \tilde{\beta})=\mathcal{N}(\phi)-\frac{M_{1}}{2}(V(\phi)-\tilde{\alpha})+\frac{3 \epsilon M_{2}}{2 \sqrt{2}}(A(\phi)-\tilde{\beta}) f(\phi)
$$

where $\tilde{\alpha}$ and $\tilde{\beta}$ are two undetermined constant parameters. Formally, we get a map between these two parameters and the solution $\phi^{n+1}$ of (35) at $t_{n+1}$. We look for $\tilde{\alpha}$ and $\tilde{\beta}$ in (35) such that the solution $\phi^{n+1}$ of (34) at time $t_{n+1}$ maintains the constraints

$$
v(\tilde{\alpha}, \tilde{\beta}):=V\left(\phi^{n+1}\right)=\alpha_{0}, \quad a(\tilde{\alpha}, \tilde{\beta}):=A\left(\phi^{n+1}\right)=\beta_{0}
$$

(up to the required accuracy) which gives a nonlinear algebraic system for $\tilde{\alpha}$ and $\tilde{\beta}$.

Borrowing the idea of the classic ALM approach, we can make use of the stabilized ETDRK method as before to repeatedly solve (34), then update the Lagrange multipliers (equivalent to (31)) by

$$
\tilde{\alpha} \leftarrow \tilde{\alpha}-\left(V\left(\phi^{n+1}\right)-\alpha_{0}\right), \quad \tilde{\beta} \leftarrow \tilde{\beta}-\left(A\left(\phi^{n+1}\right)-\beta_{0}\right),
$$

until $\tilde{\alpha}$ and $\tilde{\beta}$ converges.

Different from the pure penalty method, the ALM approach could allow the use of smaller penalty parameters $M_{1}$ and $M_{2}$ to significantly decrease the stiffness of the system (34). Thus we can use larger time step sizes in the ETD method while still having good numerical stability. Nevertheless, the update (37) behaves like a fixed point iteration and often converges slowly. A better updating process can be derived from a Newton or quasi-Newton type linearization. At the $k$-th iteration, we have

$$
\left(\begin{array}{c}
v\left(\tilde{\alpha}_{(k)}, \tilde{\beta}_{(k)}\right) \\
a\left(\tilde{\alpha}_{(k)}, \tilde{\beta}_{(k)}\right)
\end{array}\right) \approx\left(\begin{array}{c}
v\left(\tilde{\alpha}_{(k-1)}, \tilde{\beta}_{(k-1)}\right) \\
a\left(\tilde{\alpha}_{(k-1)}, \tilde{\beta}_{(k-1)}\right)
\end{array}\right)+\mathbf{T}_{(k-1)}\left(\begin{array}{c}
\tilde{\alpha}_{(k)}-\tilde{\alpha}_{(k-1)} \\
\tilde{\beta}_{(k)}-\tilde{\beta}_{(k-1)}
\end{array}\right)
$$

where $\mathbf{T}_{(k-1)}$ is the $2 \times 2$ Jacobian of $(v, a)$ at $\left(\tilde{\alpha}_{(k-1)}, \tilde{\beta}_{(k-1)}\right)$, that can be approximated via different techniques. By setting $v\left(\tilde{\alpha}_{(k)}, \tilde{\beta}_{(k)}\right)=\alpha_{0}$ and $a\left(\tilde{\alpha}_{(k)}, \tilde{\beta}_{(k)}\right)=\beta_{0}$, we have

$$
\left(\begin{array}{c}
\tilde{\alpha}_{(k)} \\
\tilde{\beta}_{(k)}
\end{array}\right) \approx\left(\begin{array}{c}
\tilde{\alpha}_{(k-1)} \\
\tilde{\beta}_{(k-1)}
\end{array}\right)-\mathbf{T}_{(k-1)}^{-1}\left(\begin{array}{c}
v\left(\tilde{\alpha}_{(k-1)}^{n}, \tilde{\beta}_{(k-1)}^{n}\right)-\alpha_{0} \\
a\left(\tilde{\alpha}_{(k-1)}^{n}, \tilde{\beta}_{(k-1)}^{n}\right)-\beta_{0}
\end{array}\right) .
$$


In another words, instead of (37), we can use the following more efficient way

$$
\left(\begin{array}{c}
\tilde{\alpha} \\
\tilde{\beta}
\end{array}\right) \leftarrow\left(\begin{array}{c}
\tilde{\alpha} \\
\tilde{\beta}
\end{array}\right)-\mathbf{T}^{-1}\left(\begin{array}{c}
V\left(\phi^{n+1}\right)-\alpha_{0} \\
A\left(\phi^{n+1}\right)-\beta_{0}
\end{array}\right)
$$

to update $\tilde{\alpha}$ and $\tilde{\beta}$. It is easy to see that the update formula (37) is actually a variant of (39), i.e., fixing the matrix $\mathbf{T}^{-1}$ as the identity matrix $\mathbf{I}$. Next we present our algorithm for solving the phase field Willmore dynamics with fixed volume and surface constraints using the stabilized ETDRK method with modified ALM approach in the following:

Algorithm 2 (Stabilized ETDRK method with modified ALM for constrained phase field Willmore dynamics). Input: the rectangular domain $\Omega \in \mathbb{R}^{3}$, a spatial grid with sizes $h_{x}$, $h_{y}$ and $h_{z}$ respectively in each dimension, the initial configuration $\boldsymbol{\Phi}_{0}$, the end time $T>0$, the target volume $\alpha_{0}=V\left(\Phi_{0}\right)$ and surface area $\beta_{0}=A\left(\Phi_{0}\right)$, the accuracy order $q$, the time step sizes $\left\{\Delta t_{n}\right\}_{n=0}^{N_{t}-1}$ such that $\sum_{n=0}^{N_{t}-1}=T$, two constant penalty parameters $M_{1}, M_{2}>0$, and a small tolerance tol $>0$.

1. Set $\tilde{\alpha}=\alpha_{0}, \tilde{\beta}=\beta_{0}$, and $\mathbf{T}^{-1}=\mathbf{I}$;

2. $\mathbf{U}^{0}=\operatorname{FFT}\left(\boldsymbol{\Phi}^{0}\right)$;

3. for $n=0,1, \cdots, N_{t}-1$ do

for $i=1,2,3$ do

Update $(\tilde{\alpha}, \tilde{\beta})$ by $(39)$;

Compute $\mathbf{U}^{n+1}=\operatorname{ETD} q \operatorname{RK}\left(\mathbf{U}^{n}, \Delta t_{n}, \mathcal{L}, \mathcal{N}_{A M}\right)$ and $\boldsymbol{\Phi}^{n+1}=\operatorname{iFFT}\left(\mathbf{U}^{n+1}\right)$;

if $\max \left(\left|V\left(\boldsymbol{\Phi}^{n+1}\right)-\alpha_{0}\right| / \alpha_{0},\left|A\left(\boldsymbol{\Phi}^{n+1}\right)-\beta_{0}\right| / \beta_{0}\right) \leq$ tol, break the for loop for next $n$;

$\operatorname{Set}\left(\tilde{\alpha}_{i}, \tilde{\beta}_{i}\right)=(\tilde{\alpha}, \tilde{\beta})$ and $\left(v_{i}, a_{i}\right)=\left(V\left(\boldsymbol{\Phi}^{n+1}\right), A\left(\boldsymbol{\Phi}^{n+1}\right)\right)$;

\section{end}

while true do

Set

$$
\mathbf{T}^{-1}=\left(\begin{array}{cc}
\tilde{\alpha}_{2}-\tilde{\alpha}_{1} & \tilde{\alpha}_{3}-\tilde{\alpha}_{1} \\
\tilde{\beta}_{2}-\tilde{\beta}_{1} & \tilde{\beta}_{3}-\tilde{\beta}_{1}
\end{array}\right)\left(\begin{array}{cc}
v_{2}-v_{1} & v_{3}-v_{1} \\
a_{2}-a_{1} & a_{3}-a_{1}
\end{array}\right)^{-1}
$$

Update $(\tilde{\alpha}, \tilde{\beta})$ by $(39)$;

Compute $\mathbf{U}^{n+1}=\operatorname{ETD} q \operatorname{RK}\left(\mathbf{U}^{n}, \Delta t_{n}, \mathcal{L}, \mathcal{N}_{A M}\right)$ and $\boldsymbol{\Phi}^{n+1}=\operatorname{iFFT}\left(\mathbf{U}^{n+1}\right)$;

if $\max \left(\left|V\left(\boldsymbol{\Phi}^{n+1}\right)-\alpha_{0}\right| / \alpha_{0},\left|A\left(\boldsymbol{\Phi}^{n+1}\right)-\beta_{0}\right| / \beta_{0}\right) \leq$ tol, break the while loop for next $n$.

$\operatorname{Set}\left(\tilde{\alpha}_{1}, \tilde{\beta}_{1}\right)=\left(\tilde{\alpha}_{2}, \tilde{\beta}_{2}\right),\left(\tilde{\alpha}_{2}, \tilde{\beta}_{2}\right)=\left(\tilde{\alpha}_{3}, \tilde{\beta}_{3}\right),\left(\tilde{\alpha}_{3}, \tilde{\beta}_{3}\right)=(\tilde{\alpha}, \tilde{\beta})$;

Set $\left(v_{1}, a_{1}\right)=\left(v_{2}, a_{2}\right),\left(v_{2}, a_{2}\right)=\left(v_{3}, a_{3}\right)$ and $\left(v_{3}, a_{3}\right)=\left(V\left(\boldsymbol{\Phi}^{n+1}\right), A\left(\boldsymbol{\Phi}^{n+1}\right)\right)$;

end

end 
In Algorithm 2, at each time step, the matrix $\mathbf{T}^{-1}$ is initialized from the result of the previous time step, then updated via the iteration. The approximate Jacobian inverse is obtained via a difference approximation (40) to make the implementation simple. One can see that if $\left(\tilde{\alpha}_{i}, \tilde{\beta}_{i}\right)$ approximate the solution $(\tilde{\alpha}, \tilde{\beta})$ of $(36)$, then by Taylor expansion, we have

$$
\mathbf{T}^{-1} \approx\left(\begin{array}{cc}
\tilde{\alpha}_{2}-\tilde{\alpha}_{1} & \tilde{\alpha}_{3}-\tilde{\alpha}_{1} \\
\tilde{\beta}_{2}-\tilde{\beta}_{1} & \tilde{\beta}_{3}-\tilde{\beta}_{1}
\end{array}\right)\left(\left(\begin{array}{cc}
v_{\alpha} & v_{\beta} \\
a_{\alpha} & a_{\beta}
\end{array}\right)\left(\begin{array}{cc}
\tilde{\alpha}_{2}-\tilde{\alpha}_{1} & \tilde{\alpha}_{3}-\tilde{\alpha}_{1} \\
\tilde{\beta}_{2}-\tilde{\beta}_{1} & \tilde{\beta}_{3}-\tilde{\beta}_{1}
\end{array}\right)\right)^{-1}=\left(\begin{array}{cc}
v_{\alpha} & v_{\beta} \\
a_{\alpha} & a_{\beta}
\end{array}\right)^{-1},
$$

thus the iteration (39) is essentially a quasi-Newton approach to accelerate the classic ALM iterations. It works effectively in practice as demonstrated in our numerical experiments.

\subsection{Tests of phase field Willmore dynamics with constraints}

We now solve the phase field Willmore dynamics with fixed volume and surface area constraints. We again took the same initial configuration (23) of two close-by spheres, the uniform spatial mesh $128^{3}$ on the spatial domain $[-\pi, \pi]^{3}$ and $\epsilon=3 h$ as in previous example given in Section 2.4.1. We have the initial volume $\alpha_{0} \approx 6.4908$ and the initial surface area $\beta_{0} \approx 19.7637$ (Note that the initial volume and surface area are numerically calculated based on the initial phase field function defined by (23) on the mesh).

We first tested the ETDRK schemes with penalty formulation for comparison purpose. The two constant penalty parameters were set to be $M_{1}=M_{2}=10$. Figure 6 shows the transformation process of these two close-by spheres with fixed volume and surface area constraints, obtained by the stabilized ETD4RK-penalty formulation scheme with $\Delta t=10^{-3}$ : the two spheres first merge together and then gradually change to the capsule shape. Figure 7 presents plots of evolutions of the energy, volume and surface area simulated by the stabilized ETD4RK scheme with some different time step sizes $\Delta t=10^{-2}, 10^{-3}, 10^{-4}$ respectively. From the Figure 7 -(a), it is easy to see that the evolution curve of the energy with the time step size $\Delta t=10^{-3}$ is nearly the same as that of $\Delta t=10^{-4}$, but is significantly different from that of $\Delta t=10^{-2}$. This observation is similar to that in the previous unconstrained phase field Willmore dynamics example. The volume and surface area curves also show same behavior. However, the volume gradually increases from around 6.49 to 7.11 (about 10\% change) and the surface area from 19.76 to 19.43 (around $1.6 \%$ change), thus violating the volume and surface area constraints. To lower the errors of volume by 100 times down to $0.1 \%$, we need to increase the penalty coefficients $M_{1}$ by 100 times to 1000 , while to lower the error of surface area down to $0.1 \%$ we need to increase $M_{2}$ by 20 times to 200 . With such larger penalty coefficients, the ETD4RK scheme with the penalty formulation blows up for $\Delta t=10^{-3}$. We then did some further tests and find that it is necessary to have $\Delta t$ down to $10^{-4}$ to maintain numerical stability of the ETD4RK scheme. This significantly increased the computational cost.

As proposed in Section 3.2, instead of increasing penalty coefficients, we can fix $M_{1}=M_{2}=10$, but adjust the values of $\tilde{\alpha}$ and $\tilde{\beta}$ so that the volume and surface area constraints can be enforced at each time step. Figure 8 presents the shape transformation process obtained by Algorithm 2, the stabilized ETD4RK scheme with modified ALM approach where the tolerance was set to be $t o l=10^{-5}$ and the time step size is $\Delta t=10^{-3}$. It is easy to notice the visible differences between the two simulations results shown in Figure 8 and Figure 6, in particular, the final state simulated by the ETD4RK scheme with modified ALM approach is a peanut shape instead of a capsule one. Figure 9 gives the corresponding plot of evolution of energy, volume and surface area. We observe that the elastic bending energy keeps decreasing and the scheme is very stable along all time. The volume and surface area are accurately maintained along the time as guaranteed by Algorithm 2 


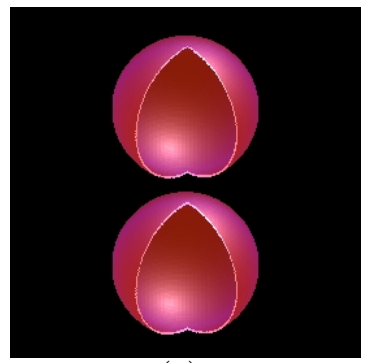

(a)

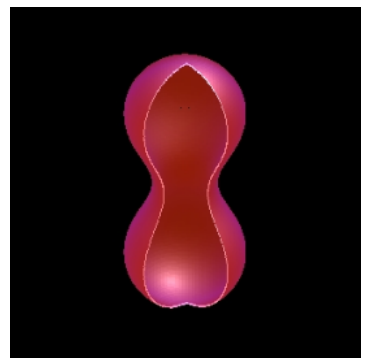

(d)

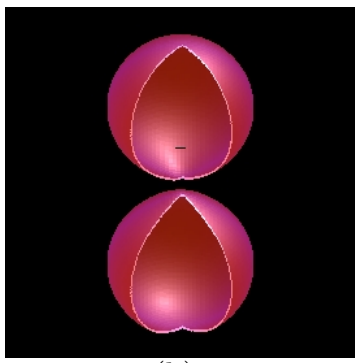

(b)

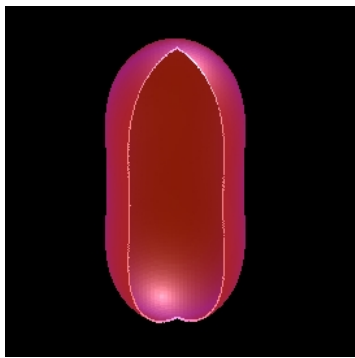

(e)

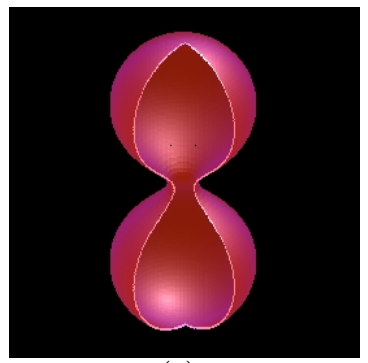

(c)

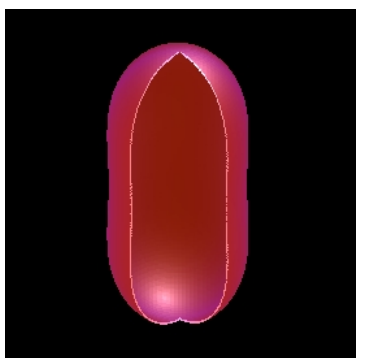

(f)

Figure 6: Simulation of the phase field Willmore dynamics of two close-by spheres (23) with fixed total volume and surface area constraints by using the stabilized ETD4RK scheme with penalty formulation $\left(M_{1}=M_{2}=10\right)$ and $\Delta t=$ $10^{-3}$. From (a) to (e): the transformed shape under the constrained Willmore fow at time $t=0,0.01,0.02,0.1,2.0,6.0$, respectively.

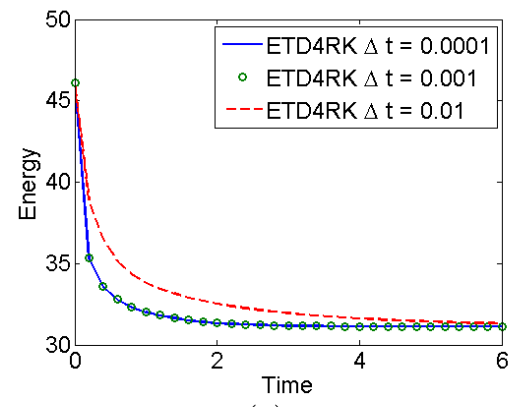

(a)

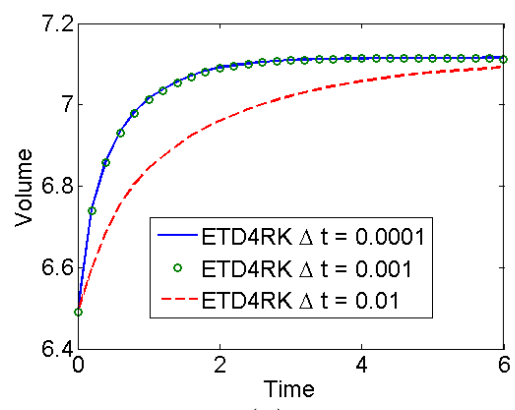

(b)

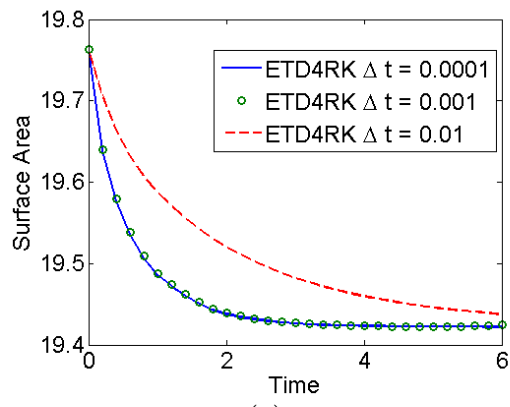

(c)

Figure 7: Plots of evolution of energy, volume and surface area for the phase field Willmore dynamics of two close-by spheres (23) with fixed volume and surface area constraints obtained by the stabilized ETD4RK scheme with penalty formulation $\left(M_{1}=M_{2}=10\right)$ and different time step sizes $\Delta t=0.01,0.001,0.0001$.

with the use of small tolerance. We also observe that for tol $=10^{-5}$ only a few (one to four) iterations of modified augmented Lagrange multiplier process are needed at most of the time steps.

We next performed the tests for convergence rates of the stabilized ETD1RK, ETD2RK and ETD4RK schemes with the modified ALM approach. We again took the small time interval [0, 0.1] as the tests in Section 2.4, and set $M_{1}=M_{2}=10$ with a smaller tolerance tol $=10^{-7}$. All results at the time $t=0.1$ are reported in Table 3 where the approximate solution produced by the ETD4RK scheme with $\Delta t=10^{-5}$ is used as the approximate exact solution (the benchmark energy is 38.493548626334) to evaluate errors of other numerical solutions. In Figure 10, we plot 


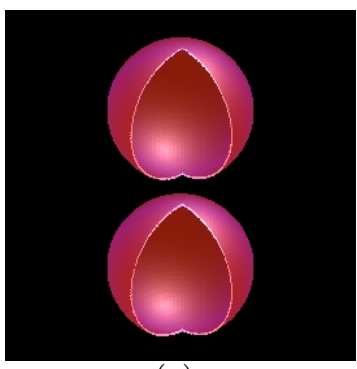

(a)

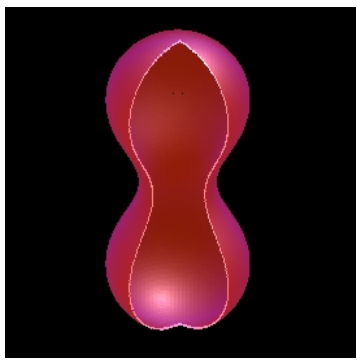

(d)

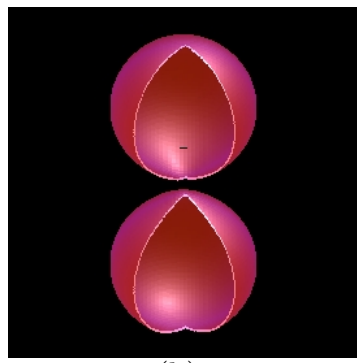

(b)

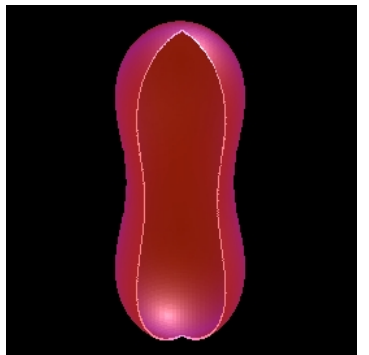

(e)

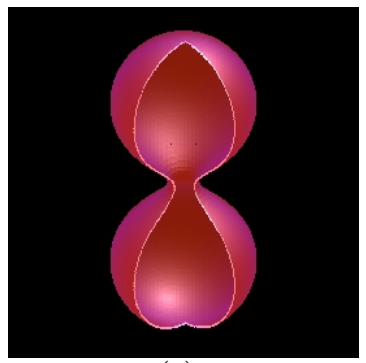

(c)

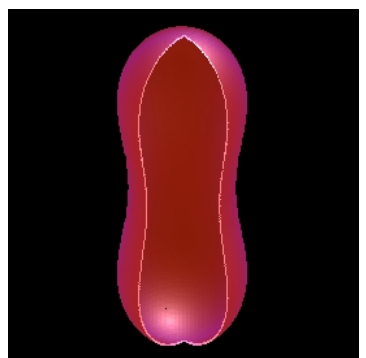

(f)

Figure 8: Simulation of the phase field Willmore dynamics of two close-by spheres (23) with fixed total volume and surface area constraints by using the stabilized ETD4RK scheme with augmented Lagrange multiplier approach $\left(M_{1}=M_{2}=10\right.$, tol $\left.=10^{-5}\right)$ with $\Delta t=10^{-3}$. From (a) to (e): the transformed shape in cut view under the constrained Willmore fow at time $t=0,0.01,0.02,0.1,2.0,6.0$, respectively.

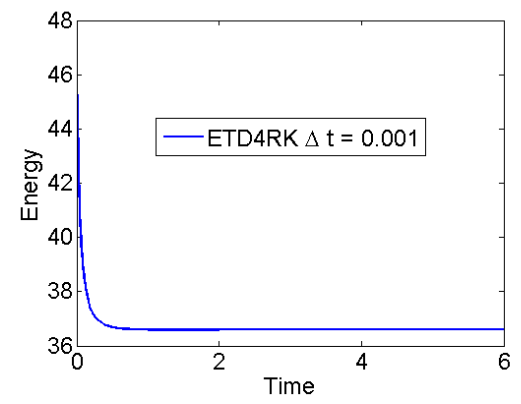

(a)

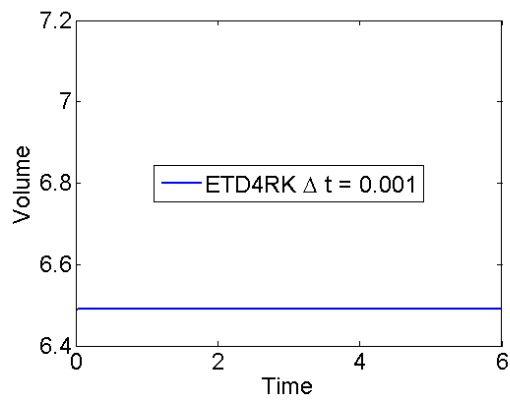

(b)

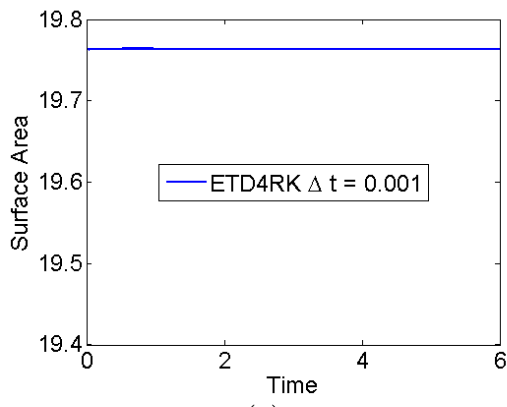

(c)

Figure 9: Plots of evolution of energy, volume and surface area for the phase field Willmore dynamics of two closeby spheres (23) with fixed volume and surface area constraints obtained by the stabilized ETD4RK scheme with augmented Lagrange multiplier approach $\left(M_{1}=M_{2}=10\right.$, tol $\left.=10^{-5}\right)$ with $\Delta t=10^{-3}$.

the time step sizes against errors. The convergence rates of all schemes are similar to those for the unconstrained Willmore problem reported in Table 1 and gradually approach their expected theoretical values.

In order to demonstrate the equivalence of the penalty formulation with very large penalty parameters and the augmented Lagrange multiplier approach, we compared the simulation results at time $t=0.1$ produced by the ETDRK4 scheme with penalty formulation with $M_{1}=M_{2}=$ 10000, 1000, 100 respectively and by the ETDRK4 scheme with modified ALM approach with $M_{1}=$ $M_{2}=10$ and $t o l=10^{-7}$, see Table 4 . The time step size $\Delta t=3.125 \times 10^{-5}$ was used for all 


\begin{tabular}{|c||c|c|c||c|c|c||c|c|c|}
\hline \multicolumn{1}{|c||}{} & \multicolumn{3}{c||}{ ETD4RK } & \multicolumn{3}{c||}{ ETD2RK } & \multicolumn{3}{c|}{ ETD1RK } \\
\hline$\Delta t$ & Energy & Error & Rate & Energy & Error & Rate & Energy & Error & Rate \\
\hline \hline $4.000 \times 10^{-3}$ & 39.39522 & 0.9016716 & - & 41.87805 & 3.3845063 & - & 43.53625 & 5.0427023 & - \\
\hline $2.000 \times 10^{-3}$ & 38.74521 & 0.2516646 & 1.84 & 40.24811 & 1.7545656 & 0.95 & 41.78343 & 3.2898822 & 0.62 \\
\hline $1.000 \times 10^{-3}$ & 38.54813 & 0.0545803 & 2.21 & 39.22792 & 0.7343695 & 1.26 & 40.32219 & 1.8286366 & 0.85 \\
\hline $5.000 \times 10^{-4}$ & 38.50347 & 0.0099230 & 2.46 & 38.75798 & 0.2644352 & 1.47 & 39.42587 & 0.9323221 & 0.97 \\
\hline $2.500 \times 10^{-4}$ & 38.49510 & 0.0015534 & 2.68 & 38.57919 & 0.0856384 & 1.63 & 38.95395 & 0.4603995 & 1.02 \\
\hline $1.250 \times 10^{-4}$ & 38.49375 & 0.0002062 & 2.91 & 38.51919 & 0.0256441 & 1.74 & 38.72014 & 0.2265926 & 1.02 \\
\hline $6.250 \times 10^{-5}$ & 38.49357 & 0.0000244 & 3.08 & 38.50079 & 0.0072370 & 1.83 & 38.60559 & 0.1120408 & 1.02 \\
\hline $3.125 \times 10^{-5}$ & 38.49355 & 0.0000024 & 3.34 & 38.49547 & 0.0019241 & 1.91 & 38.54943 & 0.0561581 & 1.00 \\
\hline
\end{tabular}

Table 3: The energies, errors and corresponding convergence rates at time $t=0.1$ by the stabilized ETD1RK, ETD2RK and ETD4RK schemes with augmented Lagrange multiplier approach for the phase field Willmore dynamics of two close-by spheres (23) with fixed volume and surface area constraints.

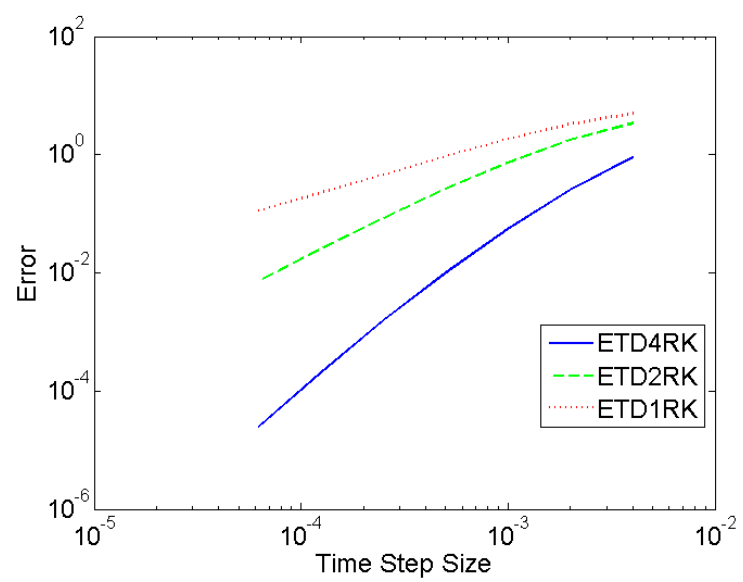

Figure 10: Logrithmic plot of energy errors vs. time step sizes of the stabilized ETD1RK, ETD2RK and ETD4RK schemes with augmented Lagrange multiplier approach for the phase field Willmore dynamics of two close-by spheres (23) with fixed volume and surface area constraints.

cases. Figure 11 gives the plots of energy evolutions produced by the penalty formulation with $M_{1}=M_{2}=10000$ and the augmented Lagrange multiplier approach. We can clearly see the convergence of the penalty formulation to the augmented Lagrange multiplier approach along the increasing of the parameters $M_{1}$ and $M_{2}$.

\begin{tabular}{|c||c|c|c||c|}
\hline \multicolumn{1}{|c||}{} & \multicolumn{3}{c||}{ Penalty Formulation } & Modified ALM \\
\hline \hline$M_{1}, M_{2}$ & 100 & 1000 & 10000 & 10 \\
\hline Energy at $t=0.1$ & 38.2263 & 38.4602 & 38.4902 & 38.4936 \\
\hline
\end{tabular}

Table 4: Comparison of the energy at $t=0.1$ simulated by the ETD4RK with penalty formulation and with modified augmented Lagrange multiplier approach. 


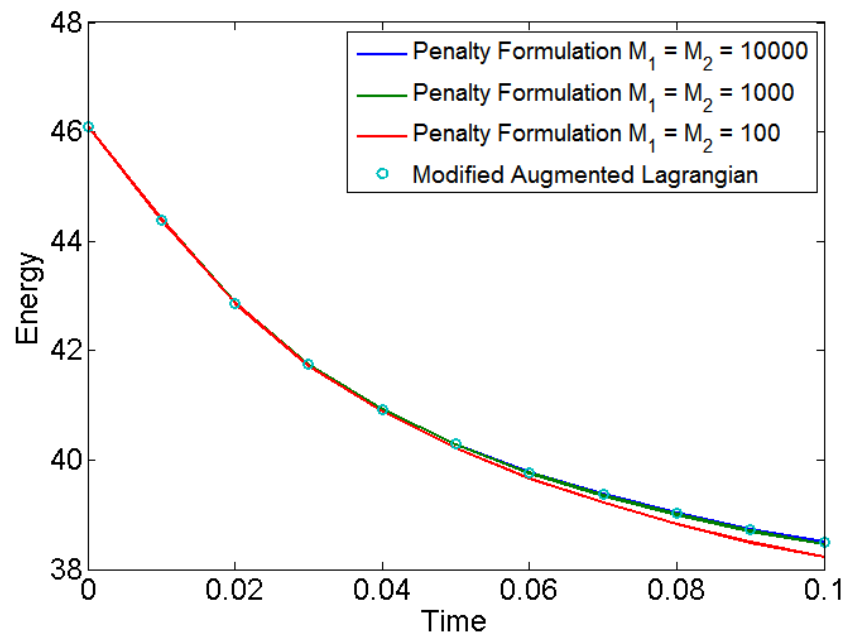

Figure 11: Comparison of energy evolution by the stabilized ETD4RK scheme with penalty formulation and with modified augmented Lagrange multiplier approach.

\section{Further application: simulation of triply-periodical minimum surfaces}

From previous discussion, we know that for the minimizer of (2) without any constraints, the zero level set of the phase field solution is an approximation of the minimum surface (with constant mean curvature 0 ). Consequently the proposed methods also can be used to compute various shapes such as the triply-periodical minimum surfaces (periodical in all three coordinate directions). Let us start from the following initial configuration (see Figure 12-(a)):

$$
\phi(x, y, z)=\left\{\begin{aligned}
1.0, & \text { if } \cos (x) \sin (y)+\cos (y) \sin (z)+\cos (z) \sin (x)>0 \\
-1.0, & \text { otherwise }
\end{aligned}\right.
$$

and try to find the corresponding triply-periodical minimum surface. We used the mesh of $128^{3}$, and chose $\epsilon=3 h$ and the time step size $\Delta t=10^{-3}$. After only 30 steps (at $t=0.03$ ) using the stabilized ETD4RK scheme, the elastic bending energy decreased from $2.99 \times 10^{6}$ to $5.08 \times 10^{-2}$, and it further decreased to $1.43 \times 10^{-8}$ at $t=5.0$. Figure 12 -(b) presents an illustration of the final approximate minimal surface in this case.

Next we start from another initial configuration (see Figure 13-(a)):

$$
\phi(x, y, z)=\left\{\begin{aligned}
1.0, & \text { if } \sqrt{x^{2}+y^{2}}<\left(\frac{\pi}{4}\right)^{2} \quad \text { or } \quad \sqrt{x^{2}+z^{2}}<\left(\frac{\pi}{4}\right)^{2} \quad \text { or } \quad \sqrt{y^{2}+z^{2}}<\left(\frac{\pi}{4}\right)^{2} \\
-1.0, & \text { otherwise. }
\end{aligned}\right.
$$

We used a larger time step size $\Delta t=10^{-2}$ in the ETD4RK scheme for this example. We observed that the elastic bending energy decreased to 0.13141 at $t=100.0$, and continued to go down to around $10^{-8}$ at $t=650.0$, see Figure 13-(b) for an illustration of the final approximate minimal surface.

\section{Discussion and conclusions}

In this paper, we have developed fast, stable and accurate numerical methods for the phase field elastic bending energy model, without or with fixed volume and surface area constraints. 


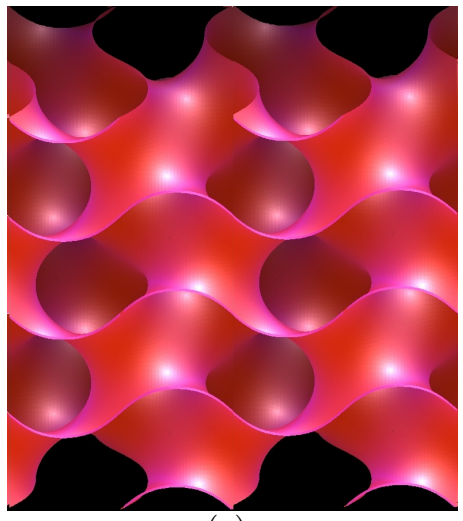

(a)

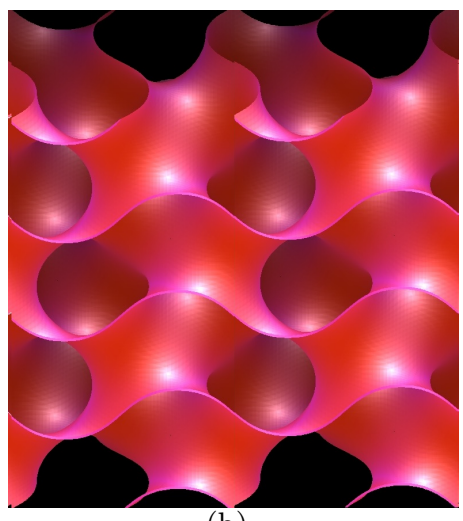

(b)

Figure 12: Simulations of the triply-periodical minimum surface starting from the initial configuration (41). (a) the initial surface and (b) the final minimum surface at $t=5.0$.

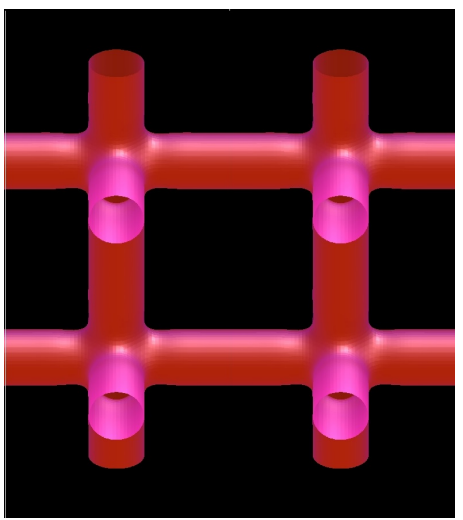

(a)

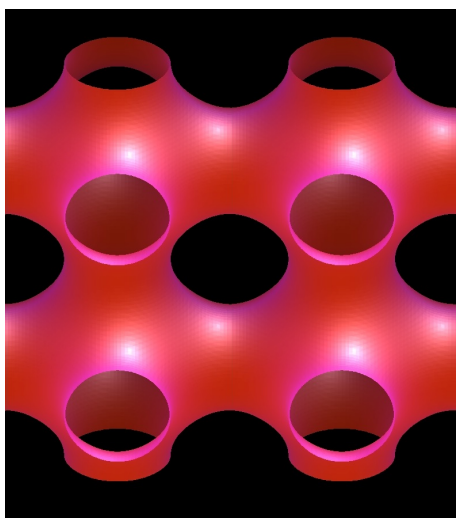

(b)

Figure 13: Simulations of the triply-periodical minimum surface starting from the initial configuration (42). (a) the initial surface and (b) the final minimum surface at $t=650.0$.

The proposed algorithms combine the explicit exponential time differencing Runge-Kutta schemes and stable linear operator splitting techniques with spectral discretizations and fast discrete Fourier transforms on regular meshes. We also design a modified Lagrange multiplier approach to efficiently and effectively deal with the constraints. Various numerical experiments demonstrate excellent performance of the proposed methods for simulating both unconstrained and constrained phase field dynamics.

One natural issue associated with the newly developed algorithm is the choice of optimal time step sizes for fast convergence to the equilibrium for energy minimization when maintaining relatively good accuracy in the dynamics. We present some numerical experiments here for a demonstration. We again took (42) as the initial configuration of the shape as in the previous section for the unconstrained phase field dynamics. We first ran the stabilized ETD4RK scheme with $\Delta t=10^{-2}$ to reach $t=0.05$, then we tested the decreased amount of energy in a single time step with different time step sizes ranging from $2.5 \times 10^{-4}$ to $10^{2}$. The ETD4RK scheme worked very stably for all tested time step sizes, and the plot of the decreased amount of energy vs. the time 
step size is shown in Figure 14-(a). It is easy to see that the larger the time step size is, the larger amount of energy decreasing we get. On the other hand, there is clearly an "elbow" phenomenon appearing in the curve, which means very large time step sizes increase computation errors but do not provide effective reduction of the energy. We did similar tests at the time $t=50.0$ and Figure 14-(b) presents the plot of the decreased amount of energy against the time step size for this case. These two curves are similar in shape, and their elbow points are both within $\left[5 \times 10^{-3}, 5 \times 10^{-2}\right]$, leading to the possibility of an optimal step size for energy reduction and dynamic accuracy.

We also repeated all tests for the stabilized ETD2RK scheme and the corresponding plots are given at Figure 15. Similar results as those for the ETD4RK scheme can be drawn except that the amount of energy reduction produced by the ETD4RK scheme is always around 3 times of that by the ETD2RK scheme for all tested time step sizes. Since the ETD4RK's cost only doubles that of the ETD2RK's, overall the ETD4RK is clearly a better choice in terms of efficiency and accuracy in practice.

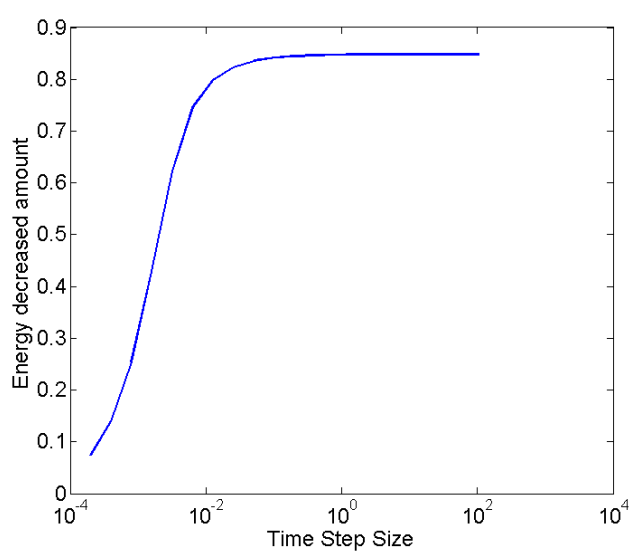

(a)

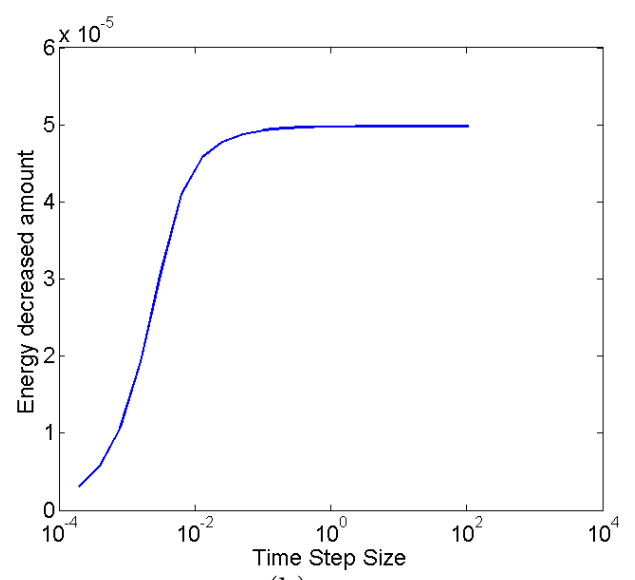

(b)

Figure 14: Plot of the decreased amount of energy vs. the time step size by the stabilized ETD4RK scheme in a single time step for the unconstrained phase field dynamics. (a) at $t=0.05$; (b) at $t=50.0$.

There are naturally many other interesting issues to be explored in the future. For example, on the theoretical side, the investigation of stability of the proposed ETDRK schemes remains largely open. Convergence of the iterations in the modified ALM approach can also be investigated. On the modeling side, we have mainly focused on the mean curvature contributions to the bending energy. To help the understanding of complex vesicle membrane morphology in applications, one may also consider extensions to situations involving the spontaneous curvature and the Gaussian curvature. More studies also can be done for high-order discretizations of phase field formulations of mutli-component vesicles and coupled vesicle-fluid interaction models.

\section{Acknowledgements}

The authors would like to thank the reviewers for their valuable comments that helped to significantly improve the paper. 


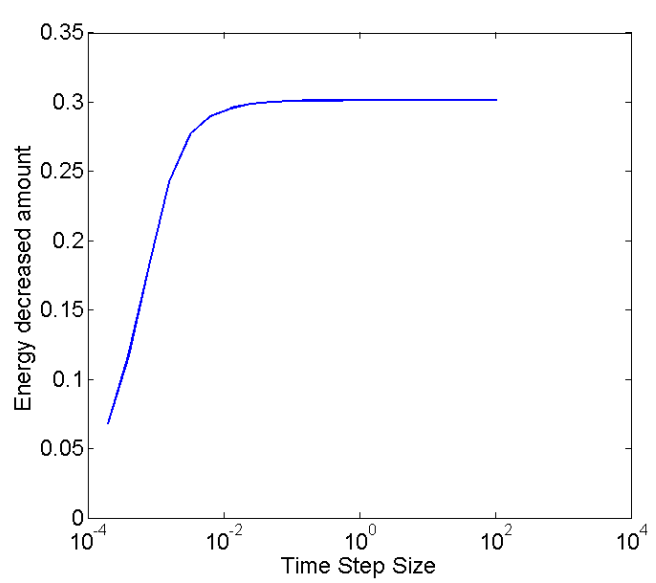

(a)

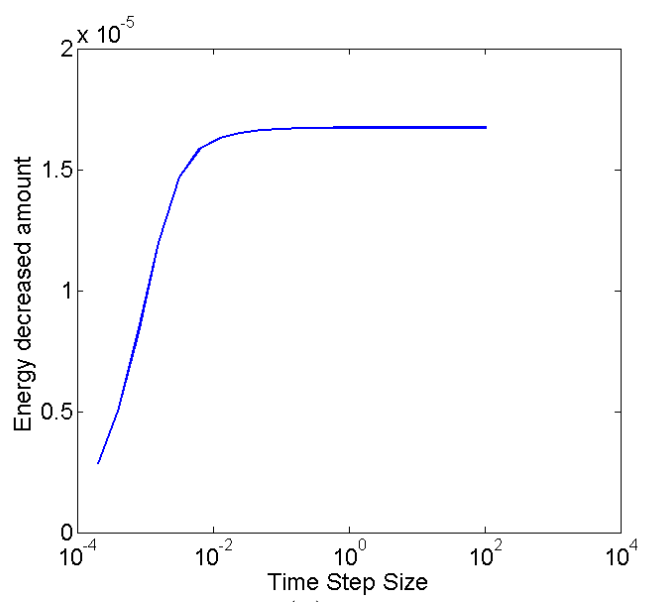

(b)

Figure 15: Plot of the decreased amount of energy vs. the time step size by the stabilized ETD2RK scheme in a single time step for the unconstrained phase field dynamics. (a) at $t=0.05$; (b) at $t=50.0$.

\section{References}

[1] G. Bellettini and L. Mugnai, Approximation of Helfrich's Functional via Diffuse Interfaces, SIAM J. Math. Anal., 42, 2010, pp. 2402-2433.

[2] E. Bretin, S. Masnou And E. Oudet, Phase-field approximations of the Willmore functional and flow, Numerische Mathematik, 131, 2015, pp. 115-171.

[3] P.B. Canham, The minimum energy of bending as a possible explanation of the biconcave shape of the human red blood cell, J. Theor. Biol., 26, 1970, pp. 61-76.

[4] R.S. Chadwick, Axisymmetric indentation of a thin incompressible elastic layer, SIAM J. Appl. Math., 62, 2002, pp.1520-1530.

[5] L.-Q. Chen And J. Shen, Applications of semi-implicit Fourier-spectral method to phase field equations, Comput. Phys. Comm., 108, 1998, pp. 147-158.

[6] S. Cox and P. Matthews, Exponential time differencing for stiff systems, J. Comput. Phys., 176, 2002, pp. 430-455.

[7] E. De Giongi, Frontiere orientate di misura minima. Editrice Tecnico Scientifica, Pisa, Italy, 1961.

[8] M. Droske and M. Rumpf, A level set formulation for Willmore flow, Interfaces Free Bound., 6, 2004, pp. 361-378.

[9] Q. Du, Phase field calculus, curvature-dependent energies, and vesicle membranes, Philosophical Magazine, 91, 2011, pp. 165-181.

[10] Q. Du, C. Liu, R. Ryham and X. Wang, A phase field formulation of the Willmore problem, Nonlinearity, 18, 2005, pp. 1249-1267. 
[11] Q. Du, C. Liu, R. Ryham And X. Wang, Modeling the spontaneous curvature effects in static cell membrane deformations by a phase field formulation, Comm. Pure Appl. Anal., 4, 2005, pp. 537-548.

[12] Q. Du, C. Liu, R. Ryham and X. Wang, Diffuse interface energies capturing the Euler number: relaxation and renomalization, Comm. Math. Sci., 5, 2007, pp.233-242.

[13] Q. Du, C. Liu, R. Ryham and X. Wang, Energetic variational approaches in modeling vesicle and fluid interactions, Physica D, 238, 2009, pp. 923-930.

[14] Q. Du, C. LiU And X. Wang, A phase field approach in the numerical study of the elastic bending energy for vesicle membranes, J. Comput. Phys., 198, 2004, pp. 450-468.

[15] Q. Du, C. LiU And X. Wang, Retrieving topological information for phase field models, SIAM J. Appl. Math., 65, 2005, pp. 1913-1932.

[16] Q. Du, C. LiU And X. Wang, Simulating the deformation of vesicle membranes under elastic bending energy in three dimensions, J. Comput. Phys., 212, 2006, pp. 757-777.

[17] Q. DU AND X. WANG, Convergence analysis of the numerical approximation to a phase model for membrane vesicle deformations, Inter. J. Numer. Anal. Model., 4, 2007, pp. 441-459.

[18] Q. Du And J. Zhang, Adaptive finite element method for a phase field bending elasticity model of vesicle membrane deformations, SIAM J. Sci. Comput., 30, 2008, pp. 1634-1657.

[19] Q. Du And W. Zhu, Analysis and applications of the exponential time differencing schemes, BIT Numer. Math.., 45, 2005, pp. 307-328.

[20] Q. Du And L. Zhu, Analysis of a mixed finite element method for phase field elastic bending energy of vesicle membrane deformation, J. Comput. Math., 24, 2006, pp. 265-280.

[21] S. Esedoglu, A. RÄTZ And M. RöGer, Colliding interfaces in old and new diffuse-interface approximations of Willmore-flow, Comm. Math. Sci., 12, 2014, pp. 125-147.

[22] A. Evans, Bending resistance and chemically induced moments in membrane bilayers, Biophys. J., 14, 1974, pp. 923-931.

[23] M. Farshbaf-Shaker And H. Garcke, Thermodynamically consistent higher order phase field Navier-Stokes models with applications to biological membranes, Dis. Cont. Dyn. Syst. $S, 4,2011$, pp. 371-389.

[24] C. Funkhouser, F. Solis and K. Thorton, Coupled composition-deformation phase-field method for multicomponent lipid membranes, Phys. Rev. E, 76, 2007, pp. 011912.

[25] R. Gu, X. Wang and M. Gunzburger, Simulating vesicle substrate adhesion using two phase field functions, J. Comp. Phy., 275, 2014, pp. 626-641.

[26] R. Gu, X. Wang and M. Gunzburger, A two phase field model for tracking vesicle-vesicle adhesion, J. Math. Bio., submitted, 2015.

[27] W. Helfrich, Elastic properties of lipid bilayers: Theory and possible experiments, Z. Naturforsch. Teil C, 28, 1973, pp. 693-703. 
[28] M. Hestenes, Multiplier and gradient methods, J. Opti. Theo. Appl., 4, 1969, pp. 303-320.

[29] D. Jamet And C. Misbah, Towards a thermodynamically consistent picture of the phase-field model of vesicles: Local membrane incompressibility., Phys. Rev. E, 76.5, (2007): 051907.

[30] L. Ju, J. Zhang AND Q. DU, FFast and accurate algorithms for simulating coarsening dynamics of Cahn-Hilliard equations, Comput. Mat. Sci., 108, pp. 272-282, 2015.

[31] L. Ju, J. Zhang, L. Zhu And Q. Du, Fast explicit integration factor methods for semilinear parabolic equations, J. Sci. Comput., 62, pp. 431-455, 2015.

[32] A. Kassam and L. Trefethen, Fourth-order time-stepping for stiff PDEs, SIAM J. Sci. Comput., 26, 2005, pp. 1214-1233.

[33] C.V. LoAn, Computational Frameworks for the Fast Fourier Transform, SIAM, 1992.

[34] P. Loreti And R. March, Propagation of fronts in a nonlinear fourth order equation, Euro. J. Appl. Math., 11, 2000, pp. 203-213.

[35] J. Lowengrub, A. Ratz and A. Voigt, Phase-field modeling of the dynamics of multicomponent vesicles: Spinodal decomposition, coarsening, budding, and fission, Phys. Rev. E, 79, 2009, pp. 031926.

[36] Y. Nagase and Y. Tonegawa, A singular perturbation problem with integral curvature bound, Hiroshima Math. J., 37, 2007, pp. 455-489.

[37] M. Röger And R. Schätzle, On a modified conjecture of De Giorgi. Mathematische Zeitschrift, 254(4), 2006, pp. 675-714.

[38] J.S. Sohn, Y.H. Tseng, S.W. Li, A. Voigt and J. Lowengrub, Dynamics of multicomponent vesicles in a viscous fluid, J. Comput. Phys., 229, 2010, pp. 119-144.

[39] X. Wang, Astmptotic Analysis of Phase Field Formulations Of Bending Elasticity Models, SIAM J. Math. Anal., 39, 2008, pp. 1367-1401.

[40] X. Wang And Q. Du, Modelling and simulations of multi-component lipid membranes and open membranes via diffusive interface approaches, J. Math. Bio., 56, 2008, pp.347-371.

[41] T.J. Willmore, A survey on Willmore immersions, Geometry and Topology of Submanifolds IV, pp. 11-166, World Science Publication, 1992.

[42] C. Xu And T. TAng, Stability analysis of large time-stepping methods for epitaxial growth models, SIAM J. Numer. Anal., 44, 2006, pp. 1759-1779.

[43] X. Yang, J. Feng, C. Liu and J. Shen, Numerical simulations of jet pinching-off and drop formation using an energetic variational phase-field method, J. Comput. Phys., 218, 2007, pp. 417-428.

[44] J. Zhang, S. Das And Q. Du, A phase field model for vesicle-substrate adhesion, J. Comput. Phys., 228, 2009, pp. 7837-7849.

[45] L. ZHU AND Q. DU, Error estimates for approximations of a gradient dynamics for phase field elastic bending energy of vesicle membrane deformation, Math. Meth. Appl. Sci., 37, 2014, pp. 913-930. 